$7-2003$

\title{
Equilibrium Impact of Value-At-Risk Regulation
}

\author{
Markus Leippold \\ University of Zurich \\ Fabio Trojani \\ University of Southern Switzerland \\ Paolo Vanini \\ University of Southern Switzerland
}

Follow this and additional works at: https://fordham.bepress.com/crif_seminar_series

Part of the Finance and Financial Management Commons

\section{Recommended Citation}

Leippold, Markus; Trojani, Fabio; and Vanini, Paolo, "Equilibrium Impact of Value-At-Risk Regulation" (2003). CRIF Seminar series. 29.

https://fordham.bepress.com/crif_seminar_series/29 


\title{
Equilibrium Impact of Value-At-Risk Regulation *
}

\author{
Markus Leippold ${ }^{\dagger}$ \\ University of Zürich \\ Fabio Trojani \\ University of Southern Switzerland \\ Paolo Vanini \\ University of Southern Switzerland
}

This Version: July 14, 2003

First Version: December 14, 2001

${ }^{*}$ The authors wish to thank seminar participants at the University of Zürich, the Swiss Federal Institute of Technology Zürich, the University of Southern Switzerland, the Bendheim Center of Finance at Princeton University, Humboldt Universität zu Berlin, the QMF 2002 Conference in Cairns, the 9th Symposium on Finance, Banking, and Insurance in Karlsruhe. In particular, we thank Giovanni Barone-Adesi, Simona Cain, Ernst Eberlein, Daniel Egloff, Damir Filipovic, Rajna Gibson, and Michel Habib for many valuable comments. Financial support by the National Center of Competence in Research "Financial Valuation and Risk Management" is gratefully acknowledged. The National Centers in Research are managed by the Swiss National Science Foundation on behalf of the federal authorities.

${ }^{\dagger}$ Correspondence information: Markus Leippold, Swiss Banking Institute, University of Zürich, Plattenstrasse 14, 8032 Zürich, Switzerland, tel: (01) 63439 62, mailto:leippold@isb.unizh.ch 


\title{
Equilibrium Impact of Value-at-Risk Regulation
}

\author{
Abstract \\ We study the partial and general equilibrium implications of value-at-risk (VaR) \\ regulation in continuous-time economies with intermediate expenditure, stochastic \\ opportunity set, and heterogeneous attitudes to risk. Our findings show that because \\ of an anticipatory effect of VaR constraints on the optimal hedging demand, the \\ partial equilibrium incentives of $\mathrm{VaR}$ regulation can lead banks to increase their \\ risk exposure in high-volatility states. In general equilibrium, VaR constraints can \\ produce unambiguously lower interest rates and higher equity Sharpe ratios. The \\ VaR impact on equity volatility and equity expected returns is ambiguous. \\ JEL Classification Codes: G11, G12, G28, D92, C60, C61. \\ Key Words: Value-at-Risk, Stochastic Opportunity Set, Regulatory Policy, Dy- \\ namic Financial Equilibria, Perturbation Theory.
}


In this paper we analyze the impact of market risk regulation on banks' optimal portfolio policies and on financial markets. We examine the impact of value-at-risk (VaR) regulation, its partial equilibrium incentives, and the general equilibrium asset-pricing implications.

Ideally, regulation aims at maintaining and improving the safety of the financial industry by defining minimal capital standards. In 1996 VaR-based risk management had already emerged as common market practice. In the 1996 Amendment on market risk regulation, the Bank of International Settlement (BIS) chose VaR as the regulatory reporting tool and is about to extend this practice to credit risk.

A thorough analysis of VaR-based regulation and its impact on financial markets is a challenging problem, since we must derive not only the bank's behavior, but also the impacts on the financial market, within a "realistic" model. We believe that such an analysis rests on at least three minimal requirements.

First, we want to base our model on a stochastic opportunity set that allows for stochastic volatility and for several forms of realistic intertemporal relations between Sharpe ratios and volatilities. If we were to assume only normally distributed asset returns, this assumption would be insufficient for analyzing the role of regulation when market conditions worsen and market volatilities increase. It is in these situations that regulation must prove its effectiveness, since VaR constraints are most likely to become binding.

Second, to take investment horizon effects into consideration, we believe that banks should solve an intertemporal optimization problem under a properly defined VaR constraint. By "properly defined," we mean that the VaR constraint should mimic the regulator's specification. We do not aim at constructing a normative theory on how banks should use VaR or some other risk measure to manage their risk exposure. Instead, we adopt an external view on risk management and take the current regulatory framework as exogenously given. 
Third, we propose that individual risk management behavior be embedded within a general equilibrium analysis with heterogeneous banks. In such a setup, the VaR constraints are endogenously determined, which allows to study the feedback effects of VaR regulation. The restriction to a homogeneous and myopic economy misses a broad set of relevant equilibrium feedback effects.

We set our requirements in a continuous-time model in which VaR-regulated investors derive utility from both intermediate expenditure and terminal wealth. We analyze equilibrium policies and prices in an economy with a stochastic investment opportunity set and heterogeneous attitudes to risk. Thus, both interest rates and the drift and volatility of asset price processes depend on a stochastic state variable.

Incorporating these features is crucial to a full understanding of the regulatory incentives and the policy implications of VaR regulation. Indeed, strengthening one of these two assumptions to either a constant opportunity set or a representative agent economy drastically simplifies, and in some cases trivializes, the emerging regulatory effects.

By applying approximate solutions to a broad class of realistic models, we find that the general economic implications of VaR regulation are very complex and cannot be easily characterized, regardless of the given economic setting. As a consequence, the intuition implied by exact solutions of particular models may be too limited for understanding the general economic consequences of VaR regulation. Hence, using asymptotic analysis in the study of VaR regulation is a necessary compromise for studying the arising equilibrium effects analytically, both under general conditions on the economy's structure and in the presence of endogenous VaR constraints. However, we note that despite the approximative nature of our approach, our comparative statics are exact.

Our main results are as follows. First, the partial equilibrium incentives of VaR regulation 
are ambiguous. Indeed, they can be quite perverse, depending on the structure of the given opportunity set dynamics and the resulting intertemporal hedging behavior. We characterize and provide explicit examples in which such intertemporal hedging behavior leads banks to increase their risk exposure in high-volatility states. Second, the endogenous general equilibrium impact of VaR regulation on equity volatility and expected returns is ambiguous, since it may bind investors with heterogeneous risk aversions in many ways. Third, VaR regulation produces unambiguous effects on interest rates and equity Sharpe ratios. Interest rates decrease and Sharpe ratios increase. In this respect, the general equilibrium impact of VaR regulation rationalizes some well-known puzzles in the financial literature (see, e.g., Cochrane (1997)). From our analysis we conclude that a statement about the effectiveness of regulation strongly depends on market factors, regulatory control variables, the market participants, and the chosen model. Given these results, the current regulatory framework and its aim to improve the safety of the financial industry, regardless of the state-dependent structure of the economy, seems highly questionable.

The paper is structured as follows. Section 1 presents some background information on previous endeavors to incorporate VaR-restricted portfolio allocation within partial and general equilibria. Section 2 presents the basic model setup and specifies the VaR constraint. Section 3 elaborates on the bank's optimization problem in the presence of VaR constraints in partial equilibrium. Section 4 investigates the general equilibrium with VaR-constrained investors and derives the implications for interest rates, the dynamics of the price of equity, and the implied portfolio policies. Section 5 concludes. 


\section{Background}

Surprisingly, there is very little academic literature that provides a thorough analysis of both the partial equilibrium incentives and general equilibrium impacts of VaR regulation in a dynamic setting. The partial equilibrium has been studied in Basak and Shapiro (2001), Cuoco, He, and Issaenko (2001), and, more recently, by Cuoco and Liu (2002). All these papers are based on a constant opportunity set assumption. To our knowledge, only two contributions have explicitly addressed a general equilibrium setting: Basak and Shapiro (2001) and Danielsson, Shin, and Zigrand (2001).

Basak and Shapiro (2001) show that investors are induced to take on a larger risk exposure than in the unconstrained setting, thereby deepening and prolonging market downturns. They ascribe this unappealing effect to the non-coherency of VaR. For a definition of coherent risk measures, see Artzner, Delbaen, Eber, and Heath (1999). Following Basak and Shapiro (2001), if regulators were to replace VaR by a coherent risk measure such as "expected shortfall," the unattractive effects of VaR-based regulation would disappear.

Danielsson, Shin, and Zigrand (2001) assume a simplified dynamic setting consisting of a sequence of myopic general equilibrium economies and use simulation to analyze the effects of VaR regulation. They find that, when compared with a benchmark unregulated economy, it adversely affects prices, liquidity, and volatility. However, the myopic structure of their economy eliminates by construction the effects of some key variables such as intermediate expenditure, the intertemporal hedging demand, and all investment horizon effects.

Cuoco, He, and Issaenko (2001) attribute the findings of Basak and Shapiro (2001) to their static definition of the VaR constraint. In a partial equilibrium, they derive numerical solutions showing that, when VaR limits are dynamically updated, no unappealing incentives arise. 
The partial equilibrium analysis of Cuoco and Liu (2002) incorporates both the optimal trading and the optimal VaR-reporting behavior of the financial institution. Under the assumption of normally distributed asset returns, these authors find that, using numerical procedures, VaR regulation can be very effective in curbing the risk of trading portfolios and in inducing truthful risk reporting.

Given the complexity of our model setup to study the implications of dynamic VaR regulation with both stochastic opportunity set dynamics and heterogeneities of risk attitudes, closed-form solutions for optimal policies are not available. We overcome this problem by making use of perturbation theory. Judd (1996) provides a comprehensive overview of perturbation theory and its application in economics. In the context of portfolio allocation, perturbation theory was previously applied by Kogan and Uppal (2001), and Trojani and Vanini (2002).

\section{The Model}

The financial market consists of a risky asset with price $P_{t}$ and an instantaneous risk-free moneymarket account with value $B_{t}$ at time $t$. The dynamics of $B_{t}$ and $P_{t}$ are

$$
d B_{t}=r\left(X_{t}\right) B_{t} d t, B_{0}=1
$$

with $r\left(X_{t}\right)$ the risk-free rate process, and

$$
d P_{t}=\alpha\left(X_{t}\right) P_{t} d t+\sigma\left(X_{t}\right) P_{t} d Z_{t}, P_{0}=p
$$


Drift and diffusion parameters in the asset's dynamics depend on a one-dimensional state variable $X_{t}$. The process $X_{t}$ follows an Itô diffusion

$$
d X_{t}=\mu_{X}\left(X_{t}\right) d t+\sigma_{X}\left(X_{t}\right) d Z_{t}^{X}, X_{0}=x
$$

with two Brownian motions $\left(Z_{t}, Z_{t}^{X}\right)$ having correlation $\mathbb{E}\left[d Z_{t} d Z_{t}^{X}\right]=\rho d t$. In the sequel, we split the drift of the asset price process into the short rate component $r\left(X_{t}\right)$ and a risk-premium component $\lambda\left(X_{t}\right)$, i.e. $\alpha\left(X_{t}\right)=r\left(X_{t}\right)+\lambda\left(X_{t}\right)$.

We consider a bank with a portfolio fraction $w_{t}$ of current wealth $W_{t}$ invested in the risky asset and a fraction $1-w_{t}$ invested in the riskless asset. To simplify notation, we write $w_{t}$ for $w\left(X_{t}\right)$. Thus, the bank's wealth dynamics are

$$
\frac{d W_{t}}{W_{t}}=\left(w_{t} \lambda\left(X_{t}\right)+r\left(X_{t}\right)\right) d t+w_{t} \sigma\left(X_{t}\right) d Z_{t}
$$

The regulator is supposed to define the bank's constraint on market risk by means of a VaR risk measure. Below, we define VaR:

Definition 1. The time-t regulatory VaR of a portfolio $w_{t}$ for a given $\mathbb{P}$-probability level $\nu \in$ $(0,1)$ and for a fixed time-horizon $\tau>0$ is defined by

$$
\operatorname{VaR}_{t}^{\nu, w}=\inf \left\{L \geq 0 \mid \mathbb{P}\left(W_{t}-\mathcal{W}_{t+\tau} \geq L \mid \mathcal{F}_{t}\right)<\nu\right\}
$$

where $\mathcal{W}_{t+\tau}$ is the portfolio value at time $t+\tau$ of a fixed-weight strategy with initial weight $w_{t}$ at time $t$.

For reporting purposes the time horizon $\tau$ is typically one day or ten days. Definition 1 is consistent with the VaR concept adopted by both practitioners and regulators. We note that 
we work with fixed relative portfolio weights, although the regulator requires to assume fixed absolute weights for a given holding period. Most practical implementations of VaR calculations, such as, e.g., RiskMetrics (1999), calculate VaR over a one-day holding period and scale it accordingly to obtain the ten-day VaR required for regulatory reporting. For the one-day holding period, it is typically assumed that the drift of portfolio changes is equal to zero. Under this assumption, the fixed absolute weight and the fixed relative weight assumptions are indeed equivalent (see Appendix A).

In our model, the bank's VaR is bounded at time $t$ by an exogenous limit $\overline{\mathrm{VaR}}_{t}$ for the given time horizon $\tau$. In the sequel, we work with a VaR limit $\overline{\mathrm{VaR}_{t}}$ proportional to current wealth, i.e.,

$$
\overline{\mathrm{VaR}_{t}}=\beta W_{t}, \beta \in[0,1]
$$

which, again, is a common choice among practitioners.

We note that the wealth dynamics in equation (4) depend on the stochastic opportunity set $X_{t}$. Thus, we cannot expect to obtain closed-form solutions for the bank's intertemporal decision problem in the presence of VaR constraints. Therefore, to retain analytical tractability, we approximate the VaR constraint implied by equations (5) and (6). We apply the Itô Taylor formula to define the first-order approximation,

$$
\log \mathcal{W}_{t+\tau} \approx \log \mathcal{W}_{t+\tau}^{(1)}=\log W_{t}+\left(r\left(X_{t}\right)+w_{t} \lambda\left(X_{t}\right)-\frac{1}{2} w_{t}^{2} \sigma\left(X_{t}\right)^{2}\right) \tau
$$

The accuracy of the approximation (7) is quantified by the next proposition, which holds for the general form of the Itô diffusion for $X$ given in equation (3).

Proposition 1. The approximation error of the first-order approximation $\mathcal{W}_{t+\tau}^{(1)}$ for the value 
$\mathcal{W}_{t+\tau}$ of a fixed-weight portfolio with initial weight $w_{t}$ is bounded by

$$
\mathbb{P}\left(\left|\log \mathcal{W}_{t+\tau}^{(1)}-\log \mathcal{W}_{t+\tau}\right| \geq M \mid \mathcal{F}_{t}\right) \leq \frac{1}{M} \mathbb{E}[|\mathbf{R}|]
$$

where

$$
\mathbb{E}[|\mathbf{R}|]=\left|\int_{t}^{t+\tau} \int_{t}^{s} \mathbb{E}\left[\mathcal{L} r\left(X_{u}\right)+w_{t} \mathcal{L} \lambda\left(X_{u}\right)+\frac{1}{2} w_{t}^{2} \mathcal{L} \sigma\left(X_{u}\right)^{2} \mid \mathcal{F}_{t}\right] d u d s\right|
$$

and $\mathcal{L}=\mu_{X} \frac{\partial}{\partial X}+\frac{1}{2} \sigma_{X}^{2} \frac{\partial^{2}}{\partial X^{2}}$ is the infinitesimal generator of $X$.

Proposition 1 can be interpreted as follows: $\mathbb{P}\left(\cdot \mid \mathcal{F}_{t}\right)$ is the conditional probability that the logarithmic difference between the approximated wealth and the true wealth exceeds the amount $M$ at time $t+\tau$. If we choose, say, $M=0.1$, Proposition 1 offers a bound on the probability that $\log \mathcal{W}_{t+\tau}^{(1)}$ and $\log \mathcal{W}_{t+\tau}$ differ by more than ten percent.

Table 1 illustrates the accuracy of the VaR approximation (8), where we assume a meanreverting geometric Brownian motion for the volatility process. In this case, $\mathbb{E}[|\mathbf{R}|]$ is obtained in closed form. The results are presented for two different conditioning values of the state variable, $X_{t}=1$ and $X_{t}=3$. For lower values of $X_{t}$ the approximation is even more accurate. According to Table 1 , the approximation bounds are generally very tight. For instance, the bound on the error probability for $M=0.01, X_{t}=1$ and a horizon $\tau=10$ is always below 0.01 percent. The bounds for $X_{t}=3$ are always below one percent and in many cases below 0.05 percent. Similar results hold for other specifications of the Itô process $d X_{t}$.

The quality of the approximation results suggests that we can reasonably use the VaR approximation in (7) to investigate the theoretical properties of the constrained portfolio selection problem. Moreover, market practice usually confines itself to regulatory VaR figures reported based on a conditional normal distribution. Such practice further motivates our approach. 
The advantage of using equation (7) as an approximate VaR constraint is that it implies some direct portfolio bounds on the optimal policy of the VaR-constrained bank. These bounds are given in the next proposition.

Proposition 2. To first order, the constraint $\operatorname{VaR}_{t}^{\nu, w} \leq \overline{V_{a R}}$ is equivalent to the following upper and lower bounds on the fraction $w_{t}$ of wealth invested in the risky asset,

$$
w_{b}^{-}\left(X_{t}\right) \leq w_{t} \leq w_{b}^{+}\left(X_{t}\right)
$$

where

$$
\begin{aligned}
w_{b}^{ \pm}\left(X_{t}\right)= & \frac{\lambda\left(X_{t}\right)}{\sigma\left(X_{t}\right)^{2}}+\frac{v}{\sigma\left(X_{t}\right) \sqrt{\tau}} \\
& \pm \frac{\sqrt{\left(\lambda\left(X_{t}\right) \tau+\sigma\left(X_{t}\right) \sqrt{\tau} v\right)^{2}+2 \sigma\left(X_{t}\right)^{2} \tau\left(r\left(X_{t}\right) \tau-\log (1-\beta)\right)}}{\sigma\left(X_{t}\right)^{2} \tau}
\end{aligned}
$$

with $v=N^{-1}(\nu)$ the $\nu$-quantile of the standard normal distribution.

The functional form (6) for the VaR limit implies a bound on the optimal portfolio fraction that is wealth-independent. Other forms of the VaR limit generally lead to wealth-dependent VaR boundaries under the above approximation procedure.

Equation (10) shows that we have $w_{b}^{+}\left(X_{t}\right) \geq 0$, and $w_{b}^{-}\left(X_{t}\right) \leq 0$ for any $X$. These inequalities hold for all functional forms $\lambda\left(X_{t}\right)$ and $\sigma\left(X_{t}\right)$. We also observe that the portfolio bounds (9) are functions of interest rates $r\left(X_{t}\right)$, and equity expected returns and volatilities $\alpha\left(X_{t}\right)$ and $\sigma\left(X_{t}\right)$. Therefore, in a general equilibrium analysis of VaR regulation, the relevant portfolio constraints must be determined in equilibrium, together with all the other equilibrium quantities. 


\section{Partial Equilibrium}

To define the bank's partial equilibrium optimization problem under VaR constraints, we start from the following assumption on preferences.

Assumption 1. The bank derives utility from final wealth $W_{T}$ according to a CRRA-utility function

$$
u(W)=\frac{W^{\gamma}-1}{\gamma}, \gamma<1
$$

According to Assumption 1, the bank derives utility from terminal wealth only. Because here we are interested in the partial equilibrium impact of VaR constraints in the presence of a stochastic opportunity set, we impose some parametric assumptions.

Assumption 2. $X_{t}$ follows a mean reverting process given by

$$
d X_{t}=\left(\theta-\kappa X_{t}\right) d t+\sigma_{X} X_{t}^{m} d Z_{t}^{X}
$$

where $\kappa, \theta, \sigma_{X}, m$ are positive constants. In the sequel we assume that the model parameters are chosen to ensure that $X_{t}$ is a strictly positive process. The risk premium, the risky asset volatility and the interest rate processes are of the form $\lambda\left(X_{t}\right)=\lambda X_{t}^{n_{1}}, \sigma\left(X_{t}\right)=\sigma X_{t}^{n_{2}}$, and $r\left(X_{t}\right)=r$, where $\lambda, n_{1}, n_{2}, r$ are non-negative constants.

We note that under these assumption we still cover a broad range of diffusion-based asset pricing models, such as those recently explored by Anderson, Benzoni, and Lund (2002). Furthermore, we must carefully select the parameters $n_{1}$ and $n_{2}$ to avoid trivial settings in which VaR constraints are either vacuous or binding a priori. We discuss this issue in Appendix B.

To address the bank's optimization problem, we base the regulatory VaR constraint on 
the approximate VaR constraint implied by Proposition 1. Denoting by $\mathrm{BR}(W, X)$ the budget constraints (3) and (4), and by $\mathcal{C}(X)$ the approximate VaR constraint (10), the optimization problem becomes

$$
(\mathbf{P} 1): \quad J(W, X, t)=\max _{w \in \mathcal{R}(W, X)} \mathbb{E}\left[u(W) \mid \mathcal{F}_{t}\right]
$$

where $\mathcal{R}(W, X)=\{w \in \mathrm{BR}(W, X)\} \cap \mathcal{C}(X)$. Intuitively, the solution of problem (P1) must provide optimal investment strategies characterized by a region where the VaR constraint binds and a region where it does not bind.

Proposition 3. Consider the control problem $(\mathbf{P} 1)$ under the Assumptions 1 and 2. In a region where the J-function is increasing and jointly strictly concave, the optimal portfolio policy is

$$
w^{*}\left(X_{t}, t\right)= \begin{cases}w_{b}^{+}\left(X_{t}\right), & \text { if } w_{f}\left(X_{t}\right) \geq w_{b}^{+}\left(X_{t}\right), \\ w_{b}^{-}\left(X_{t}\right), & \text { if } w_{f}\left(X_{t}\right) \leq w_{b}^{-}\left(X_{t}\right), \\ w_{f}\left(X_{t}, t\right), & \text { else },\end{cases}
$$

where

$$
w_{f}\left(X_{t}, t\right)=-X_{t}^{n_{1}-2 n_{2}} \frac{\lambda J_{W}}{\sigma^{2} W J_{W W}}-\rho X_{t}^{m-n_{2}} \frac{\sigma_{X} J_{W X}}{\sigma W J_{W W}}
$$

and $w_{b}^{ \pm}\left(X_{t}\right)$ is given in equation (10).

\subsection{Perturbation in Risk Aversion}

Apparently, inserting the optimal policies of Proposition 3 back into the Hamilton-JacobiBellman (HJB) equation for problem (P1) given in equation (D.12), Appendix D, leads to a highly nonlinear optimality condition for the value function. Therefore, we apply an asymptotic analysis that computes the value function and the implied optimal policies as a power series in 
$\gamma$. From the homogeneity properties of problem $(\mathbf{P} 1)$, the value function $J$ must take the form

$$
J(W, X, t)=\frac{e^{\gamma g(X, t)} W^{\gamma}-1}{\gamma}
$$

for some unknown function $g(X, t)$. Expanding $g(X, t)$ in the parameter $\gamma$, i.e.,

$$
g(X, t)=g_{0}(X, t)+\gamma g_{1}(X, t)+\frac{1}{2} \gamma^{2} g_{2}(X, t)+O\left(\gamma^{3}\right)
$$

the optimal policy up to first order in $\gamma$ is follows from (15), (16), and (17) as

$$
w_{f}^{(1)}\left(X_{t}, t\right)=\underbrace{(1+\gamma) X_{t}^{n_{1}-2 n_{2}} \frac{\lambda}{\sigma^{2}}}_{(A)}+\underbrace{\gamma X_{t}^{m-n_{2}} \frac{\rho \sigma_{X}}{\sigma} \frac{\partial g_{0}(X, t)}{\partial X}}_{(B)}
$$

Therefore, if $g_{0}(X, t)$ can be determined, the first-order policy of an investor with risk aversion $1-\gamma$ is also determined. Equation (18) gives the approximate optimal policy as a sum of two approximate subpolicies, a myopic portfolio, (A), and an intertemporal hedging position, (B). Obviously, the magnitude and direction of these quantities depend critically on the choices of $m, n_{1}$, and $n_{2}$.

In general, given a stochastic opportunity set dynamics, it is not possible to explicitly determine the optimal intertemporal hedging policy. Therefore, we apply perturbation theory to analyze the asymptotic impact of VaR constraints for a broad range of model dynamics in partial and general equilibrium. In the perturbation approach, the problem of characterizing the first-order intertemporal hedging demand requires calculating the function $g_{0}$. By construction, $g_{0}$ is fully determined as soon as we can compute the value function of a log-utility investor. 
Proposition 4. Consider the optimization problem (P1). The function $g_{0}$ in (18) is equal to

$$
g_{0}(X, t)=g_{0}^{f}(X, t)+g_{0}^{c}(X, t)
$$

where $g_{0}^{f}$ is the corresponding $g_{0}$-function in an unconstrained economy, and $g_{0}^{c}$ is an add-on factor induced by the presence of VaR-regulation. The explicit formulas are provided in the Appendix D, equations (D.25) and (D.26).

When we examine Proposition 4, we see that the impact of VaR regulation is only through the intertemporal hedging component. This hedging component is additive-separable, which allows us to make some clear-cut statements about the first-order impacts of VaR constraints on portfolio allocation.

\subsection{Convergence and Accuracy of the Perturbation Approach}

We proceed in three steps. First, we obtain higher-order corrections for the optimal policies of problem $(\mathbf{P 1})$. Second, we demonstrate that for some given reasonable a-priori bounds on the terms in the perturbation series, the whole series converges. Third, we compute different finite-order approximations and use a numerical example to illustrate the speed of convergence of the perturbation series.

\subsubsection{Convergence}

We obtain higher-order policy approximations from equations (15), (16), and (17). The $n$ thorder approximation of $w_{f}$ in Proposition 3 is given as

$$
w_{f}^{(n)}(X, t)=X^{n_{1}-2 n_{2}} \frac{\lambda}{\sigma^{2}} \frac{1-\gamma^{n+1}}{1-\gamma}+X^{m-n_{2}} \frac{\rho \sigma_{X}}{\sigma} \sum_{j=0}^{n-1} \frac{\gamma^{j+1}}{j !} \frac{\partial g_{j}(X, t)}{\partial X} \frac{1-\gamma^{n-j}}{1-\gamma}
$$


where $g_{j}$ is the $j$ th order function in the expansion of $g(X, t)$ for $\gamma$.

Corollary 1 gives a natural a priori bound on the unknown functions $g_{j}(X, t), j \in \mathbb{N}^{0}$, which ensures convergence of the perturbation theory.

Corollary 1. For the control problem $(\mathbf{P} 1)$ for $\gamma>-1$, the optimal nth-order approximation $w_{f}^{(n)}$ converges to $w_{f}$ if $\left|\partial g_{j}(X, t) / \partial X\right| \leq K X_{t}^{2 j\left(n_{1}-n_{2}\right)}, K>0, j \in \mathbb{N}^{0}$. The limit policy is bounded by

$$
\left|w_{f}(X, t)\right|=\lim _{n \rightarrow \infty}\left|w_{f}^{(n)}(X, t)\right| \leq X^{n_{1}-2 n_{2}} \frac{\lambda}{\sigma^{2}} \frac{1}{|1-\gamma|}+X^{m-n_{2}} \frac{|\rho \gamma| \sigma_{X} e^{\gamma X^{2\left(n_{1}-n_{2}\right)}} K}{\sigma|1-\gamma|} .
$$

In the sequel we focus on model settings in which convergence of the series of policy approximations $w_{f}^{n}$ can be granted. The constraint $\gamma>-1$ is not restrictive for our purposes, because we can reasonably assume that banks are less risk averse than individual investors.

\subsubsection{Accuracy}

To compute the higher-order functions $g_{j}$, we define $\log W_{T}=\log W_{t}+H_{t, T}$ for the wealth dynamics, where

$$
H_{t, T}:=\int_{t}^{T}\left(r+\lambda X_{s}^{n_{1}} w_{s}-\frac{1}{2} w_{s}^{2} \sigma^{2} X_{s}^{2 n_{2}}\right) d s+\int_{t}^{T} w_{s} \sigma X_{s}^{n_{2}} d Z_{s}
$$

Then, we expand $g(X, t)$ as a power series in $\gamma$ and insert it in the equality

$$
\mathbb{E}\left[\frac{W_{T}^{\gamma}-1}{\gamma} \mid \mathcal{F}_{t}\right]=\frac{e^{\gamma g(X)} W_{t}^{\gamma}-1}{\gamma}
$$

Finally, we expand the LHS and the RHS of equation (23) as a power series in $\gamma$. By matching the resulting coefficient for each power of $\gamma$ in the resulting power series, we obtain the following 
characterizations for higher-order functions $g_{j}(X, t)$.

Corollary 2. For problem (P1), calculating the nth order approximation of optimal portfolio weights means considering all $(n+1)$ th-moments of the portfolio's log return.

Corollary 2 shows the direct link between the order of the $g_{j}$-functions in the perturbation series and the higher moments of the return $H_{t, T}$ on optimally invested wealth. Whenever the $n$th order of the function $g(X, t)$ enters the approximation for the optimal portfolio policy, the approximation is taking into account the $(n+1)$ th moment of $H_{t, T}$. We note that the entity $H_{t, T}$ in (22) is itself a function of $\gamma$ through the optimal policy $w_{t}$. Therefore, the computation of $g_{n}$ in Corollary 2 needs a recursive procedure that expands also the optimal policy $w_{t}$ in $(22)$ as a function of $\gamma$. To compute $g_{1}$, we need only the optimal policy of a log utility investor. By contrast, higher-order functions $g_{j}, j>2$, require a more involved recursive procedure. ${ }^{1}$

\subsubsection{Numerical Example}

To illustrate the accuracy of the perturbative solutions, we compare the finite-order policy approximations implied by Corollary 2 for $j=0,1$ with the corresponding exact portfolio policy. We do this for an unconstrained portfolio optimization and in a model setting corresponding to $n_{1}=2, n_{2}=1, r=0$, and $m=1$ in Assumption 2. We obtain exact solutions by using the Monte Carlo simulation method recently proposed by Cvitanic, Goukasian, and Zapatero (2003) and Detemple, Garcia, and Rindisbacher (2003). Both approaches are restricted to a complete market model setting. Therefore, we adapt our model accordingly and work with the following parametric specifications:

$$
\begin{aligned}
\frac{d P_{t}}{P_{t}} & =0.05 X_{t}^{2} d t+0.25 X_{t} d Z_{t} \\
d X_{t} & =\left(0.8-0.8 X_{t}\right) d t+0.2 X_{t} d Z_{t}
\end{aligned}
$$


Figure 1 illustrates our findings. Point $A$ in Figure 1 gives the log investor's optimal portfolio policy. The bold straight line in Figure 1 is the first-order approximation for the portfolio policy. The second-order approximation is represented by the bold dashed line. The circled line is the exact portfolio policy estimated by Monte Carlo simulation.

As is apparent from Figure 1, the second-order approximation has already produced very accurate results. The first-order approximation does not fit the convexity of the portfolio profile as a function of $\gamma$. However, it produces the correct sign about the direction of the effects obtained for risk-aversion parameters that differ from the log utility case.

\subsection{Incorporating Intermediate Expenditure}

Incorporating expenditure flows into the bank's optimization problem puts us back to the known portfolio problem in which utility is derived from both terminal wealth and intermediate consumption. ${ }^{2}$ To analyze the impact of VaR constraints on the optimal expenditure path of a bank, we modify Assumption 1 as follows.

Assumption 3. Banks derive utility from both an intermediate expenditure process $\left(C_{t}\right)_{0 \leq t \leq T}$ and from terminal wealth $W_{T}$. They maximize the expected value of the random variable

$$
V(W, C, t)=\int_{t}^{T} e^{-\delta s} \frac{C_{s}^{\gamma}-1}{\gamma} d s+e^{-\delta T} \frac{W_{T}^{\gamma}-1}{\gamma}, \quad 0 \leq \delta<1
$$

where $\delta \geq 0$ is the subjective discount rate.

In the presence of intermediate expenditure, wealth dynamics become

$$
d W_{t}=\left(\left(r-c_{t}\right)+w_{t} \lambda\left(X_{t}\right)\right) W_{t} d t+w_{t} \sigma\left(X_{t}\right) W_{t} d Z_{t}
$$


where $c_{t}=C_{t} / W_{t}$ is the expenditure rate. It follows from equation (27) that future current wealth in the presence of an expenditure policy is given by

$$
\log W_{T}=\log W_{t}+H_{t, T}
$$

with

$$
H_{t, T}:=\int_{t}^{T}\left(w_{s} \lambda\left(X_{s}\right)+r-c_{s}-\frac{1}{2} w_{s}^{2} \sigma\left(X_{s}\right)^{2}\right) d s+\int_{t}^{T} w_{s} \sigma\left(X_{s}\right) d Z_{s}
$$

Again, we define the relevant VaR limit as a fraction of current wealth as in equation (6). In (6) we assumed a constant portfolio $w_{t}$ over the relevant time horizon $[t, t+\tau)$. In a similar vein, we lock in the expenditure rate $c_{t}$ at the rate prevailing at time $t$. Given Assumption 2 and Proposition 2, the Itô-Taylor approximated VaR constraint in the presence of intermediate expenditure is given by

$$
Q(w, c)=r-c+\lambda X^{n_{1}} w-\frac{1}{2} w^{2} \sigma^{2} X^{2 n_{2}}-\frac{1}{\tau} \log (1-\beta)+\frac{1}{\sqrt{\tau}} v w \sigma X^{n_{2}} \leq 0
$$

For $(c, w)$, we obtain a constrained set $\mathcal{C}(X)$ given by

$$
(c, w) \in \mathcal{C}(X)=\{Q(w, c) \leq 0\}
$$

Since $Q(w, c)$ is a quadratic function of $w$ and a linear function of $c$, the set $\{(c, w): Q(w, c)=0\}$ describes a parabola in $(w, c)$-space. We obtain the portfolio fractions $w_{b}^{ \pm}$as the solutions of the equation $Q(c, w)=0$, given any feasible expenditure rate $c>0$. The convexity of the constrained set $\mathcal{C}(X)$ is ensured by the fact that $\partial^{2} Q(c, w) / \partial w^{2}<0$. 
Thus, with Assumption 3, the relevant optimization of a VaR-constrained investor reads

$$
(\mathbf{P} 2): \quad J(W, X, t)=\max _{(c, w) \in \mathcal{R}(W, X)} \mathbb{E}\left[V(W, C, t) \mid \mathcal{F}_{t}\right]
$$

where $\mathcal{C}(X)$ incorporates the presence of intermediate expenditure in the VaR constraint. ${ }^{3}$

Proposition 5. Given Assumptions 2 and 3 and the optimization problem (P2), the first-order approximations of the optimal policies are given by

$$
\begin{aligned}
w^{(1)}(X, t) & =(1+\gamma) X^{n_{1}-2 n_{2}} \frac{\lambda}{\sigma^{2}}+\gamma X^{m-n_{2}} \frac{\rho \sigma_{X}}{\sigma} \frac{\partial g_{0}(X, t)}{\partial X}, \\
c^{(1)}(X, t) & =\frac{1-\gamma\left(g_{0}(X, t)+\log A_{t}\right)}{A_{t}}
\end{aligned}
$$

where $A_{t}=e^{-\delta(T-t)}+\frac{1-e^{-\delta(T-t)}}{\delta}$ and $g_{0}(X, t)=g_{0}^{f}(X, t)+g_{0}^{c}(X, t)$. Explicit expressions for $g_{0}^{f}(X, t)$ and $g_{0}^{c}(X, t)$ are given in the Appendix D, equations (D.41) and (D.42).

Again, the function $g_{0}$ is decomposed in a term that corresponds to the solution of a problem without constraints, $g_{0}^{f}(X, t)$, and a term that reflects the marginal impact of the VaR constraint, $g_{0}^{c}(X, t)$. The latter term is associated with the utility loss that is caused by the presence of regulatory VaR constraints. It also reflects the bank's anticipations on the fact that VaR constraints might be binding in the future. This anticipation reduces the expected utility compared to an unconstrained optimal policy, and so affects the current optimal expenditure and investment decision. In particular, the existence of VaR constraints produces a non trivial anticipative effect on the bank's decision and alters the intertemporal hedging demand. This dynamic optimal behavior differs fundamentally from the one implied by a myopic portfolio optimization. ${ }^{4}$ 


\subsection{Partial Equilibrium: Do VaR Constraints Distort Incentives?}

Here, we study the partial equilibrium effects of VaR regulation for the model class defined in Assumption 2. Corollary 3 below characterizes the impact of VaR constraints on the optimal policies of problem (P2), relative to the solutions for the unconstrained portfolio problem.

Corollary 3. We satisfy Assumption 2 and 3 and consider the partial equilibrium problem (P2) with its first-order optimal policy approximations. Before VaR constraints become binding, a bank with $\gamma>0(\gamma<0)$ increases (decreases) optimal expenditure $c_{t}$; the bank's optimal portfolio is unaffected if $n_{2}=n_{1}$; the bank's optimal portfolio is unaffected if $\gamma=0$. Given $n_{1} \neq n_{2}$, the bank increases or decreases its exposure to the risky asset according to:

\begin{tabular}{|l||c|l|}
\hline & $\gamma \rho>0$ & $\gamma \rho<0$ \\
\hline \hline$\frac{\partial g_{0}}{\partial X}>\frac{\partial g_{0}^{f}}{\partial X}$ & increase & decrease \\
\hline$\frac{\partial g_{0}}{\partial X}<\frac{\partial g_{0}^{f}}{\partial X}$ & decrease & increase \\
\hline
\end{tabular}

Moreover,

$$
\frac{\partial g_{0}}{\partial X}>\frac{\partial g_{0}^{f}}{\partial X} \Leftrightarrow n_{1}<n_{2}, \quad \frac{\partial g_{0}}{\partial X}<\frac{\partial g_{0}^{f}}{\partial X} \Leftrightarrow n_{1}>n_{2},
$$

whenever

$$
v \sigma X^{n_{2}} \sqrt{\tau}+\lambda X^{n_{1}} \tau+D\left(\sigma^{2} X^{2 n_{2}} \tau-1\right) \leq 0
$$

Corollary 3 shows that the direction of an anticipative impact of VaR constraints on optimal portfolios depends on several quantities:

1) Pure preference parameters, via the risk aversion related term $\gamma$,

2) Market dynamics, via the correlation parameter $\rho$, 
3) A joint interplay of preferences, market dynamics, and regulatory parameters, via the sign of the derivative $\partial g_{0} / \partial X-\partial g_{0}^{f} / \partial X$. This sign crucially depends on the return and volatility specifications, i.e., on the parameters $n_{1}$ and $n_{2}$.

The driving force behind 1 through 3 is the impact of an anticipated binding VaR constraint in the future on today's hedging policy of a risk-averse investor. Indeed, for $\gamma=0$, we see that no anticipatory effect due to the VaR constraint is present. However, as soon as $\gamma \neq 0$ intertemporal hedging arises optimally when $n_{1} \neq n_{2}$ and the existence of VaR constraints does affect today's optimal portfolios even before the VaR constraint becomes binding.

The case $n_{1}=n_{2}$ corresponds to a situation in which Sharpe ratios are state independent. In this case, no intertemporal hedging motives emerge even for $\gamma \neq 0$. Consequently, VaR constraints do not have any anticipatory impact on investors' optimal risk exposures. The geometric Brownian motion model considered in Cuoco, He, and Issaenko (2001) and Basak and Shapiro (2001) is clearly a special case of this setting. Therefore, our results emphasize the restrictiveness of the geometric Brownian motion assumption when studying the incentive compatibility of VaR regulation.

In the absence of VaR constraints, the direction of the hedging demand depends on the impact of stochastic volatility on an investors' opportunity set. As soon as the equity Sharpe ratio, defined as $\lambda\left(X_{t}\right) / \sigma\left(X_{t}\right)$, is an increasing function of $X$, an increase in volatility yields a better opportunity set in the future, thereby implying $\partial g_{0}^{f} / \partial X>0$ in Proposition 4. By Assumption 2, this is the case as soon as $n_{1}>n_{2}$. In the case $n_{1}<n_{2}$, we typically get $\partial g_{0}^{f} / \partial X<0$, because higher levels of volatility in the future imply a worsening of tomorrow's stochastic opportunity set. Thus, depending on the relative magnitude of $n_{1}$ and $n_{2}$, high volatility is either a "good" or a "bad" state. 
When we introduce VaR constraints, their first-order effect is characterized by the difference

$$
\frac{\partial g_{0}}{\partial X}-\frac{\partial g_{0}^{f}}{\partial X}
$$

which is directly related to the marginal impact of VaR constraints on today's hedging demand. In the subsequent discussion, for simplicity we focus again on the case without expenditure in Proposition 4.

The sign of the difference in equation (37) determines the direction of the VaR-induced excess hedging demand, given a parameter choice $\gamma, \rho$. Generally, we can expect that for models in which volatility is a good (bad) state, we will have

$$
\frac{\partial g_{0}}{\partial X}-\frac{\partial g_{0}^{f}}{\partial X}>0 \quad, \quad(<0)
$$

because, when compared with the unconstrained case, VaR constraints reduce the marginal utility that derives from an optimal constrained hedging policy when compared with the unconstrained case. Under condition (36) the relation in (38) is equivalent to $n_{1}<n_{2}\left(n_{1}>n_{2}\right)$. From a practical viewpoint, when using realistic parameter choices, condition (36) holds for many explicit model settings.

Corollary 3 shows that the anticipatory effect of VaR constraints can lead to either an increase or a decrease in the bank's exposure in the risky asset, depending on the structure of the underlying opportunity set dynamics. Empirical evidence suggests that stock price movements are negatively correlated (see, e.g., Anderson, Benzoni, and Lund (2002)). Given a negative correlation $\rho$ and a risk-aversion coefficient $\gamma>0$, VaR regulation has an adverse (positive) effect on the riskiness of the bank's portfolio decision for models in which $\frac{\partial g_{0}}{\partial X}-\frac{\partial g_{0}^{f}}{\partial X}>0,(<0)$.

We note that while $\rho, \gamma$, and the difference (37) jointly determine the sign of the anticipatory 
impact of VaR regulation, the difference $m-n_{2}$ determines the absolute amount of the hedging demand, since $\partial g_{j}(X, t) / \partial X$ is scaled by $X^{m-n_{2}}$ in equation (20).

The results of Corollary 3 are further illustrated within explicit model settings. We first consider the impact of VaR regulation in a model specified by

$$
\lambda\left(X_{t}\right)=\lambda X_{t}^{2}, \quad \sigma\left(X_{t}\right)=\sigma X_{t}, \quad \sigma_{X}\left(X_{t}\right)=\sigma_{X} X_{t} .
$$

Thus, with the above specification volatility is a good state.

Figure 2 plots the optimal portfolio strategies of a VaR-constrained and a VaR-unconstrained investor for $\gamma=0.5$ as functions of volatility $\sigma\left(X_{t}\right)$. (If $\gamma<0$, the direction of the effects just changes sign.) Panels (A), (C), and (E) assume a positive, and Panels (B), (D), and (F) a negative, correlation between $X_{t}$ and risky asset returns. For a one-year investment horizon $(T=1)$, we observe in Panels (A) and (B) that the difference of the two portfolio strategies is small. However, in Panels (C) and (E) where $T=5$ and $T=10$, we see that the risk exposure of a VaR-constrained bank has already been substantially reduced before the VaR constraint becomes binding. Therefore, anticipating that the VaR constraint might become binding in the future leads to a reduction of the bank's exposure in the risky asset. As expected, when correlation is negative (see, e.g., Panel (D)), we see a reversed anticipative effect. The bank increases the optimal risk exposure before the VaR constraint becomes binding. When volatility increases further, the constraint will eventually become binding and the bank will finish with a lower risk exposure compared to an unconstrained bank.

When we use a conditionally normal dynamic VaR setting we can transform a VaR limit into an equivalent expected shortfall limit. Therefore, in our setting, imposing an expected shortfall limit is equivalent to imposing a tighter confidence bound on the VaR limit. 
Figure 3 illustrates the impact of tightening the confidence bound for model (39). Again, we distinguish between positive and negative correlation ((Panels $(A),(C)$, and (E) and (B), (D), and (F), respectively). We adopt the confidence levels $\nu=0.01$ (Panels (A) and (B)) and $\nu=0.05$ (Panels $(\mathrm{C})$ and $(\mathrm{D})$ ). As in Figure 2, we observe an increase in the bank's exposure when correlation is negative for both confidence levels. In Panels (E) and (F), we plot the differences between the constrained portfolio policies under the two confidence levels. In Panel (F) a tighter confidence level induces the bank to optimally increase its risk exposure for low and moderate volatilities. Only for volatilities larger than about 50 percent does a tighter confidence level lead to a decrease in the bank's risk exposure.

We next consider a setting in which volatility is a bad state by specifying

$$
\lambda\left(X_{t}\right)=\lambda, \sigma\left(X_{t}\right)=\sigma X_{t}, \sigma_{X}\left(X_{t}\right)=\sigma_{X} X_{t}
$$

In this model, excess expected returns are constant, implying Sharpe ratios that are inversely related to volatility. In Panels (A) and (B) of Figure 4, we plot the implied optimal policies obtained for a VaR-constrained and an unconstrained investor with $\gamma=0.5$. A striking difference from the results from model (39) is that the VaR constraint tends to become binding at low, rather than at high, volatility states $\sigma\left(X_{t}\right)$. This result derives directly from the dynamic structure of model (40), causing Sharpe ratios to be high precisely when volatility is low, and contrasts the dynamic specification (39) in which the exact opposite happens.

From a regulatory perspective, an important partial equilibrium implication of this model setting is that VaR constraints are going to limit the bank's risk exposure in low volatility states, but in high volatility states they will affect optimal exposures only via the anticipatory effect on the intertemporal hedging demand. Moreover, since in model (40) volatility is a bad state, intertemporal hedging goes in the opposite direction to that in model (39), implying 
$\frac{\partial g_{0}}{\partial X}-\frac{\partial g_{0}^{f}}{\partial X}<0$. Thus, in Corollary 3, the anticipative effect of VaR constraints goes exactly in the opposite direction of what we observe for model (39).

Depending on whether the product $\rho \gamma$ is positive (in Panel (A)) or negative (in Panel(B)), the constrained bank either decreases or increases its risk exposure relative to the unconstrained bank. The latter case implies that in the presence of VaR regulation, a large risk exposure is optimally selected precisely in the risky states of the world, i.e., when volatility is already at a high level. Clearly, this is a paradoxical partial equilibrium implication of VaR regulation in the presence of a stochastic opportunity set.

\section{General Equilibrium}

Intuitively, the aggregate effect of $\mathrm{VaR}$ regulation on individual portfolio demands will eventually influence the equilibrium dynamics of asset prices and interest rates. Thus, it also has an impact on the definition of the relevant VaR-constrained portfolios themselves. Therefore, a complete analysis of VaR regulation requires that we examine general equilibrium implications. The partial equilibrium results in Section 3 are the necessary starting point for this analysis.

Using perturbation theory, the computation of first-order general equilibria in a heterogeneous economy means determining the optimal policies of a log investor in a homogeneous economy. This procedure gives us a way to provide analytical characterizations of the general equilibrium impact of VaR regulation, and to study its dependence as a function of exogenous parameters, such as regulatory variables, by means of comparative statics. The perturbative solutions can offer economic insights and interpretation of the emerging equilibrium quantities that cannot be obtained by means of a pure numerical approach. We find that even a small deviation from the homogeneous log economy shows some substantial and significant effects on 
financial market variables, particularly on the Sharpe ratio.

\subsection{The Exchange Economy}

We consider an exchange economy with a financial market consisting of two financial instruments, an instantaneous risk-free asset and a stock. The risk-free asset is available in zero net supply and the interest rate $r_{t}$ is determined in equilibrium together with the asset price dynamics. The risky asset is a contingent claim on aggregate endowment, $e_{t}$, with dynamics

$$
\frac{d e_{t}}{e_{t}}=\mu_{e}\left(X_{t}\right) d t+\sigma_{e}\left(X_{t}\right) d Z_{t}^{e}
$$

where $Z_{t}^{e}$ is a standard Brownian motion. The state variable $X_{t}$ follows an Itô diffusion

$$
d X_{t}=\mu_{X}\left(X_{t}\right) d t+\sigma_{X}\left(X_{t}\right) d Z_{t}^{X}
$$

The instantaneous correlation between the endowment process and the state variable process is given by $\mathbb{E}\left(d Z_{t}^{e} d Z_{t}^{X}\right)=\rho_{e X}$. The economy is populated by two banks, banks $I$ and $I I$, equipped with heterogeneous risk-aversion indices $\gamma_{I}$ and $\gamma_{I I}$, respectively. Both banks solve the optimization problems $(\mathbf{P} 2)^{I}$ and $(\mathbf{P} 2)^{I I}$ according to equation (32). We denote by $\omega_{t}^{I}$ and $\omega_{t}^{I I}=1-\omega_{t}^{I}$ the cross-sectional wealth of bank $I$ and $I I$, respectively, i.e.,

$$
\omega_{t}^{i}=\frac{W_{t}^{i}}{W_{t}^{I}+W_{t}^{I I}}, i=I, I I
$$

where $W_{t}^{i}$ is the current wealth of bank $i$. We describe the general equilibrium in this economy by means of the extended state variables process $\chi_{t}=\left(X_{t}, \omega_{t}^{I}\right)^{\top}$. Hence, the stock price $P_{t}$ 
equals aggregate wealth, implying a gross return dynamics given by

$$
\begin{aligned}
\frac{d P_{t}+e_{t} d t}{P_{t}} & =\alpha(\chi, t) d t+\sigma_{P 1}(\chi, t) d Z_{t}^{X}+\sigma_{P 2}(\chi, t) d Z_{t}^{e} \\
& =\alpha(\chi, t) d t+\sigma(\chi, t)^{\top} d Z_{t}
\end{aligned}
$$

where $Z_{t}=\left(Z_{t}^{e}, Z_{t}^{X}\right)^{\top}$. The drift vector $\alpha(\chi, t)$ and the volatility vector $\sigma(\chi, t)$ must be determined in equilibrium.

Definition 2. We call a process $\left(\alpha(\chi, t), \sigma(\chi, t), r(\chi, t), w_{t}^{I}, w_{t}^{I I}, c_{t}^{I}, c_{t}^{I I}\right)$ an asymptotic pure exchange equilibrium if

a) individual portfolio rules $w_{t}^{I}, w_{t}^{I I}$ are optimal to first-order, i.e., they are of the form given in Proposition 5.

b) the financial market clears,

$$
w_{t}^{I} \omega_{t}^{I}+w_{t}^{I I} \omega_{t}^{I I}=1+O\left(\gamma^{2}\right)
$$

b) the market for consumption goods clears,

$$
c_{t}^{I} W_{t}^{I}+c_{t}^{I I} W_{t}^{I I}=e_{t}+O\left(\gamma^{2}\right)
$$

We use this equilibrium definition in the sequel to study the first-order general equilibrium effects of VaR regulation. 


\subsection{General Equilibrium Asymptotics}

The general structure of the portfolio and expenditure policies is the same as the one obtained for the partial equilibrium solutions in Proposition 5. However, the function $g_{0}$ in Proposition 5 is determined endogenously by the general equilibrium as an equilibrium function $g_{0 e}$, which depends on the extended state vector $\chi$ and the preference parameters $\gamma_{I}$ and $\gamma_{I I}$ as well. This fact motivates an expansion of $g_{0 e}$ for the risk-aversion parameters $\gamma_{I}$ and $\gamma_{I I}$ :

$$
g_{0 e}\left(\chi, \gamma_{I}, \gamma_{I I}, t\right)=g_{00}(\chi, t)+\left(\begin{array}{c}
\gamma_{I} \\
\gamma_{I I}
\end{array}\right)^{\top} g_{0,1}(\chi, t)+O\left(\gamma_{I}^{2}, \gamma_{I I}^{2}\right)
$$

In this case, the function $g_{00}$ is independent of $\gamma_{I}$ and $\gamma_{I I}$ and is uniquely determined by the value function of a log utility investor in a representative agent economy. For brevity, we write in the sequel $g_{0 e}\left(\chi, \gamma_{I}, \gamma_{I I}, t\right)=g_{0 e}(\chi, t)$, and $O\left(\gamma^{2}\right)$ for $O\left(\gamma_{I}^{2}, \gamma_{I I}^{2}\right)$. Moreover, we denote by $*=f, c$ the optimal policies of an unconstrained and a constrained investor, respectively.

We note that the general equilibrium function $g_{00}$ in a homogeneous log-utility economy is independent of the presence of VaR constraints, because the market clearing condition in such an economy restricts the equilibrium portfolio fractions to one. Moreover, $g_{00}$ depends on the state $X_{t}$ but not on cross-sectional wealth $\omega_{t}$, which is a constant in representative agents economies (see equation (C.3) in Appendix C). The first-order general equilibrium portfolio policies for bank $j, j=\{I, I I\}$, take the form

$$
\begin{aligned}
w^{j *}(\chi, t) & =\left(1+\gamma_{j}\right) \frac{\lambda^{*}(\chi, t)}{\left\|\sigma^{*}(\chi, t)\right\|^{2}}+\gamma_{j} \eta_{t}^{*}+O\left(\gamma^{2}\right), \\
c^{j *}(\chi, t) & =\frac{1}{A_{t}}-\gamma_{j} \xi_{t}^{*}+O\left(\gamma^{2}\right),
\end{aligned}
$$


where

$$
\begin{aligned}
\xi_{t} & =\frac{g_{00}\left(X_{t}, t\right)+\log A_{t}}{A_{t}}, \\
\eta_{t}^{*} & =\frac{\sigma_{P X}^{*}\left(X_{t}, t\right)}{\left\|\sigma^{*}(\chi, t)\right\|^{2}} \frac{\partial g_{00}(X, t)}{\partial X},
\end{aligned}
$$

with $\sigma_{P X}^{*}(\chi, t)$ as the covariances between stock price changes and the state variables $X$. We note that the function $\xi_{t}$ is not affected by VaR regulation to first order, and is a function of $X$ and $t$, but not of $\omega_{t}^{I}$. On the other hand, $\eta_{t}^{*}$ depends on cross-sectional wealth via $\sigma^{*}$ and $\sigma_{P X}^{*}$. To express the equilibrium asymptotics relevant for our analysis, we introduce a weighted aggregate risk-aversion coefficient $\Delta$ defined by:

$$
\Delta=\gamma_{I} \omega_{t}^{I}+\gamma_{I I}\left(1-\omega_{t}^{I}\right)
$$

Proposition 6 summarizes the properties of the first-order general equilibrium when for both banks the VaR constraint does not bind. Since, as mentioned, the anticipative impact of VaR constraints is of a higher order in general equilibrium, the first-order general equilibrium is equivalent to an economy where no VaR constraint is present.

Proposition 6. With an endowment and state variable process in equations (41) and (42), and in the absence of VaR-constraints, it follows:

1) the equilibrium interest rate is given by

$$
r^{f}(\chi, t)=\alpha^{f}(\chi, t)-\left\|\sigma^{f}(\chi, t)\right\|^{2}\left(1-\left(1+\eta_{t}^{f}\right) \Delta\right)+O\left(\gamma^{2}\right) .
$$


2) the cross-sectional wealth dynamics of investor I is given by

$$
d \omega_{t}^{I}=\left(\gamma_{I}-\gamma_{I I}\right) \omega_{t}^{I}\left(1-\omega_{t}^{I}\right)\left(\xi_{t} d t+\left(1+\eta_{t}^{f}\right) \sigma_{e}\left(X_{t}\right) d Z_{t}^{e}\right)+O\left(\gamma^{2}\right)
$$

3) drift and volatility of the cumulative return process in (44) are given by

$$
\begin{aligned}
& \alpha^{f}(\chi, t)=\delta+\mu_{e}\left(X_{t}\right)+\left(\partial_{t} A_{t}-1\right) \xi_{t} \Delta+A_{t} \Delta\left(\frac{\partial \xi_{t}}{\partial t}+\sigma_{e X} \frac{\partial \xi_{t}}{\partial X_{t}}\right)+O\left(\gamma^{2}\right), \\
& \sigma^{f}(\chi, t)=\left(\begin{array}{c}
\sigma_{P 1}^{f}(\chi, t) \\
\sigma_{P 2}^{f}(\chi, t)
\end{array}\right)=\left(\begin{array}{c}
A_{t} \Delta \frac{\partial \xi_{t}}{\partial X_{t}} \sigma_{X}\left(X_{t}\right) \\
\sigma_{e}\left(X_{t}\right)
\end{array}\right)+O\left(\gamma^{2}\right) .
\end{aligned}
$$

We note that since $d \omega_{t}^{I}$ is of order $O(\gamma)$ in an unconstrained economy, the drift and the variance of the equilibrium price process in Proposition 6 do not depend on $\omega_{t}^{I}$. On the other hand, the equilibrium interest rate $r^{f}$ depends on $\omega_{t}^{I}$ via the weighted risk-aversion coefficient $\Delta$.

We examine the general equilibrium first-order asymptotics in a VaR regulated economy for those states in which the second bank does hit the VaR constraint but the first one does not. We assume without loss of generality that $\gamma_{I}<\gamma_{I I}$. To obtain tractable expressions for the relevant quantities in the constrained economy, we perform a further expansion in the parameter $\tau$, which is typically a short time interval (e.g., a day).

Proposition 7. Given an endowment and state variable process in equations (41) and (42), and an economy where bank II is VaR-constrained and bank I is not, it follows that in states in which the VaR constraint is binding: 
i) the equilibrium interest rate is given by

$$
r^{c}(\chi, t)=\alpha^{c}(\chi, t)-\left\|\sigma^{c}(\chi, t)\right\|^{2}+\left\|\sigma^{c}(\chi, t)\right\| \frac{v\left(1-\omega_{t}^{I}\right)}{\sqrt{\omega_{t}^{I} B}}+h\left(\gamma_{I} ; \tau\right)+O\left(\gamma^{2}, \tau^{2}\right)
$$

where $B$ and $h\left(\gamma_{I} ; \tau\right)$ depend on regulatory control variables and are given in Appendix D.

ii) the cross-sectional wealth dynamics of bank I is given by

$$
d \omega_{t}^{I}=\mu_{0}^{\omega c}(\chi, t) d t+\sigma_{0}^{\omega c}(\chi, t) d Z_{t}^{e}+O(\gamma, \tau)
$$

iii) drift and volatility of the cumulative price process in (44) are given by

$$
\begin{aligned}
& \alpha^{c}(\chi, t)=\alpha^{f}(\chi, t)+\left(\gamma_{I}-\gamma_{I I}\right) a(\chi, t)+O\left(\gamma^{2}, \tau^{2}\right), \\
& \sigma^{c}(\chi, t)=\sigma^{f}(\chi, t)+\left(\gamma_{I}-\gamma_{I I}\right) \xi_{t} \sigma_{0}^{\omega c}(\chi, t)+O\left(\gamma^{2}, \tau^{2}\right),
\end{aligned}
$$

where $\alpha^{f}(\chi, t)$ and $\sigma^{f}(\chi, t)$ are given in Proposition 6. The functions $a(\chi, t), \mu_{0}^{\omega c}\left(\chi^{c}, t\right)$ and $\sigma_{0}^{\omega c}\left(\chi^{c}, t\right)$ are given in (D.72), (D.64) and (D.65).

Proposition 7 allows us to directly compare the general structure of the equilibrium impact of VaR regulation with the structure of the unconstrained economy in Proposition 6. We note that in Proposition 7, the size of the difference $\alpha^{f}-\alpha^{c}$ and $\sigma^{f}-\sigma^{c}$ in the risky asset expected return and volatility depends on quantities such as

1. the heterogeneity in risk aversions, through $\gamma_{I}-\gamma_{I I}$;

2. the investment horizon, via $A_{t}$;

3. cross-sectional wealth, via $\mu_{0}^{\omega c}$ and $\sigma_{0}^{\omega c}$; 
4. the structure of the exogenous state dynamics, i.e. the exogenous opportunity set, via $\rho_{e X}, \sigma_{X}, \sigma_{e X}$ and $\xi_{t}$

In particular, we see that a large heterogeneity in risk aversion amplifies the VaR impact on the drift and volatility of equity returns. Further, in the constrained economy, cross-sectional wealth matters to first order in determining equity dynamics, because the market clearing condition causes a nonlinear dependence of equilibrium interest rates $r^{c}$ on cross-sectional wealth. This nonlinearity is absent in the unconstrained economy.

Proposition 7 implies that a homogeneous general equilibrium economy puts strong restrictions on the way VaR regulation affects the equilibrium variables. In fact, in such economies, VaR regulation impacts only interest rates, but not the equilibrium dynamics of equity, i.e., its drift and volatility.

\subsection{General Equilibrium: Do VaR Constraints Distort the Economy?}

Here, we examine the general equilibrium implications of VaR regulation for some more explicit model settings. In particular, we consider a class $\mathcal{M}$ of model specifications given by

$$
\mathcal{M}:\left\{\begin{array}{l}
M_{0}: \quad \mu_{e}\left(X_{t}\right)=\mu_{e}, \quad \sigma_{e}\left(X_{t}\right)=\sigma_{e} X_{t}, \quad \gamma_{I}=\gamma_{I I}=0 \\
M_{1}: \mu_{e}\left(X_{t}\right)=\mu_{e}, \quad \sigma_{e}\left(X_{t}\right)=\sigma_{e} X_{t}, \quad \gamma_{I} \neq \gamma_{I I} \neq 0 \\
M_{2}: \quad \mu_{e}\left(X_{t}\right)=\mu_{e} X_{t}, \quad \sigma_{e}\left(X_{t}\right)=\sigma_{e} X_{t}, \quad \gamma_{I} \neq \gamma_{I I} \neq 0 .
\end{array}\right.
$$

We assume that the state variable $X$ follows a mean-reverting geometric Brownian motion, i.e., $m=1$ in Assumption 2. (Alternatively, we could assume a square-root or a Gaussian process for $X$. However, the results do not change qualitatively.) Model $M_{0}$ is a representative agent economy with a pure stochastic volatility model for the endowment process $e_{t}$. The representative agent is equipped with a log-utility function. Model $M_{1}$ extends model $M_{0}$ to 
account for moderate heterogeneities in risk aversion in the presence of a pure stochastic volatility model for $e_{t}$. Already, a moderate difference in risk aversion has strong effects on equilibrium variables. In the setting of model $M_{0}$, no first-order impact of VaR regulation on equity dynamics is present. By contrast, in model $M_{1}$, heterogeneity in risk aversion also affects the drift and the volatility of equity returns. Model $M_{2}$ studies the impact of VaR regulation in the presence of a mean reversion in both expected endowment returns and endowment volatility. Figures 5 through 8 summarize graphically our findings for some calibrations of models in the class $\mathcal{M}$.

\subsubsection{Model $M_{0}$}

Figure 5 presents results for model $M_{0}$ when the correlation $\rho_{e X}$ between endowment and volatility states is positive. In this economy, since drift and volatility of equity returns are unaffected, we focus on the resulting optimal portfolios, interest rates, and cross-sectional wealth dynamics. In the top left panel, because of the market clearing condition, portfolio fractions are equal to one for all states of $X_{t}$ left of point A, i.e., for "low" or "moderate" endowment volatility states (the straight line to the left of point A) . For a "high" endowment volatility, bank $I I$ hits the VaR constraint (the dotted line) and is forced to reduce its risk exposure below one (the dashed line). At the same time, to achieve market clearing, bank $I$ must increase its exposure above one (the straight line right of point A). To make such an investment more attractive, the interest rate $r$ must decrease. In the top right panels, equilibrium interest rates (the straight line) to the left of point A are on the dotted curve. This line corresponds to the equilibrium interest-rate level for the unconstrained economy. The equilibrium interest-rate level in the constrained economy is on the straight line to the right of point A. For such states of $X_{t}$, the interest-rate level is below the unconstrained economy.

The lower panels illustrate the impact of the regulation on the cross-sectional wealth dy- 
namics. In these panels, we plot the cross-sectional wealth drift and volatility as a function of $X$. To the left of point A both parameters are zero, since cross-sectional wealth is constant in the unregulated homogeneous economy. However, as soon as bank II hits the constraint, both the drift and volatility of the cross-sectional wealth dynamics increase monotonically with the underlying endowment volatility state. These changes affect the conditional variance in the interest-rate dynamics. Therefore, we conclude that within the setting of a representative agent economy of the form given in model $M_{0}$, a VaR regulation can have a destabilizing equilibrium effect, though the effect is more pronounced on money markets than on equity markets.

\subsubsection{Model $M_{1}$}

Figure 6 illustrates the results for model $M_{1}$ when we introduce heterogeneity in risk aversion within the basic setting of model $M_{0}$. Bank $I I$ is assumed to be less risk averse than bank $I$ $\left(\gamma_{I}=-0.1\right.$ and $\gamma_{I I}=0.4$, respectively). The top left panel illustrates the optimal policies of bank $I I$ (dashed line) and bank $I$ (straight line), respectively, as functions of $X$. To the left of points $\mathrm{A}$ and $\mathrm{B}$, both banks are unconstrained. Bank II invests in a leveraged risky portfolio financed in equilibrium by bank $I$, which is primarily investing in a long position in the riskless asset. As volatility increases to states $X_{t}$, corresponding to points to the right of point A, bank II hits the regulatory VaR constraint (the dotted line through point A) and is forced to reduce its risk exposure. As a consequence, Bank I reduces its equilibrium investment in the riskless asset and increases its risk exposure to equity.

We note that the VaR constraint through point $\mathrm{A}$ is determined by the equilibrium interest rate, drift, and volatility of the equity dynamics prevailing in an unconstrained economy. As soon as bank $I I$ hits point $A$, these equilibrium quantities change to their corresponding values in the constrained economy. Therefore, the relevant VaR constraint shifts immediately to the 
new equilibrium level (the dotted line below point $\mathrm{A}$ ).

This feature highlights the endogenous nature of VaR constraints in general equilibrium. The increased demand for bonds by bank II leads to a decrease in equilibrium interest rates for volatility states to the right of point A. For these particular states, equilibrium interest rates in the constrained economy (the dashed line) are below those prevailing in an unconstrained economy (the dotted line). However, a decrease in interest rates is not a sufficient incentive for bank $I$ to clear the equity excess supply of bank $I I$. Therefore, equilibrium equity expected returns and volatility also change, and both jump upwards over the given support of $X_{t}$ in the two bottom panels of Figure 6. For states in which bank $I I$ is constrained they are both higher than in the unconstrained economy. Only when the state $X$ increases further, will volatility eventually be lower than in the unconstrained economy.

Moreover, since interest rates, drift, and volatility must change in such a way that bank $I$ increases its demand for stocks (point $B$ in Figure 6), the overall effect is an increase in the implied equity Sharpe ratio. This increase is confirmed by Figure 7, which plots the implied Sharpe ratio as a function of the state $X_{t}$. At point $A$, the Sharpe ratio jumps to a higher level and obtains a steeper slope as a function of $X_{t}$ (the straight line beyond point $A$ ) than does the Sharpe ratio function in the unconstrained economy (the dotted line). Thus, the presence of heterogeneously VaR-regulated financial institutions produces relatively high Sharpe ratios, high volatilities, and low interest rates, a stylized fact that several standard financial models struggle to explain.

\subsubsection{Model $M_{2}$}

In an economy in which banks are subject to heterogeneous VaR constraints, the stylized facts obtained within model $M_{1}$ are by no means the only possible equilibrium implication. To 
emphasize this point, in Figure 8 we present the results obtained for the dynamics of model $M_{2}$ when the correlation parameter $\rho_{e X}$ is negative. In this case, because of the intertemporal hedging motive, the more risk-averse bank $I I$ is less exposed in equity (the dashed bold line in the top left panel) than bank $I$ (the straight bold line in the top left panel). Therefore, regulation constrains the less risk-averse, but also less exposed, bank II. When bank II hits the $\mathrm{VaR}$ constraint at point $\mathrm{A}$, it must reduce its risk exposure by reallocating wealth to the risky asset, thereby inducing a lower interest rate (top right panel). In equilibrium, the more risk-averse Bank I must now clear this excess supply for equity through a higher demand for equity starting from point B. Therefore, the implied Sharpe ratios prevailing in the constrained economy will be higher, just as in the previous model $M_{1}$. However, when we examine the bottom panels of Figure 6, we observe that for states in which Bank II is constrained, both the

equity expected return and the volatility are lower than in the unconstrained economy. Hence, by contrast with the results within model $M_{1}$, we observe that a heterogeneous VaR regulation lowers the aggregate volatility risk of the equity market, thereby achieving one of the tasks that VaR regulation is intended to perform.

\section{Conclusion}

In this paper we analyze the partial equilibrium incentives and the general equilibrium implications of VaR-based regulation. We do so within a continuous-time economy with stochastic opportunity set, intermediate expenditure, and heterogeneities in risk aversions. In partial equilibrium, we show that VaR-induced incentives can enhance the bank's risk exposure. However, such a distortion of incentives is totally unrelated to the lack of coherence of VaR. Instead, it depends on the optimal intertemporal hedging behavior that arises out of the dynamics of a given opportunity set and the degree of risk aversion. 
As we expected, when we move from a partial to a general equilibrium analysis makes the analysis of VaR regulation considerably more complex, since in a general equilibrium setting, risk regulation also affects the bank's optimization problem indirectly. It does so via its endogenous impact on the economy and its indirect effect on the relevant individual VaR constraints. Since VaR constraints are defined as functions of both interest rates and drift and volatilities of the stock price processes, they are determined endogenously. Therefore, in equilibrium, VaR regulation causes highly nonlinear effects and jumps in the parameters of the asset price dynamics.

In a general equilibrium, the effectiveness and possible distortion of VaR-based regulation depend on several quantities, including regulatory control variables, pure market factors, and individual investors' parameters. Therefore, the impacts of VaR regulation on equilibrium variables are very complex and hardly predictable. However, we can draw some important conclusions. First, the impact of VaR regulation depends strongly on the heterogeneity structure in risk attitudes, even if heterogeneity is moderate. Second, for unregulated institutions, an investment in a risky asset becomes more attractive, because VaR regulation tends to cause an increase in the equity Sharpe ratios. Third, an increase in the Sharpe ratio can imply either an increase or a decrease in equity expected returns and volatilities. Interest rates unambiguously decrease. Finally, when the regulated bank becomes constrained, equilibrium interest rates, asset price drift, and volatility can jump.

Our analysis shows that the optimal design of regulatory standards for market risk remains an unsolved problem and an urgent issue for future research. Since the exact effects and directions of a VaR regulation cannot be established unambiguously for a sufficiently general class of realistic models, the question arises, "Is there a regulatory standard yielding a clear statement about its economic impacts?" Since current regulation fails to answer this question, we believe 
there is an obvious need to justify the considerable costs caused to the financial industry by the implementation of regulatory standards. 


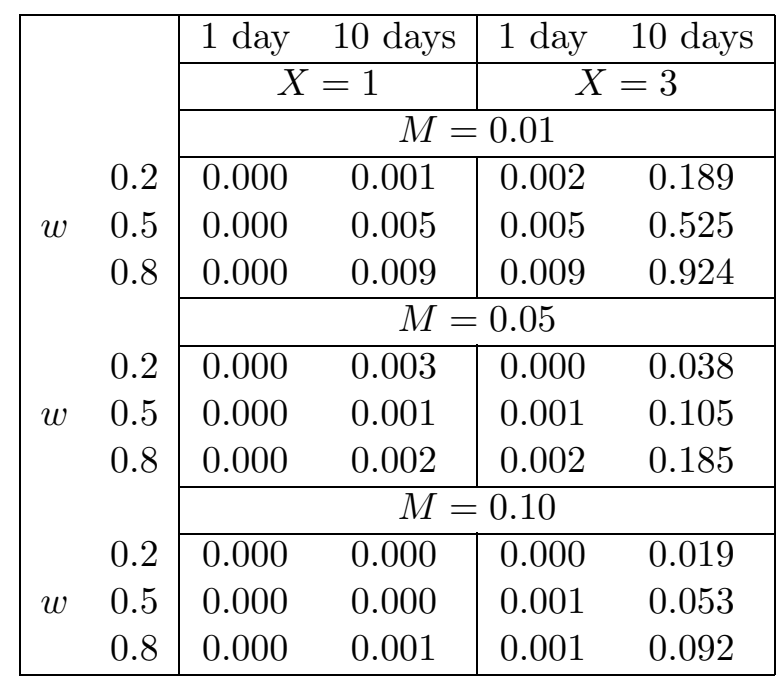

Table 1: First-order Approximation. The table displays the upper bound for the probabilities given in Proposition 1. Calculated numbers are percentage numbers. We specify our model as: $d X=\left(\theta-\kappa X_{t}\right) d t+\sigma_{X} X d Z_{t}^{X}, r\left(X_{t}\right)=r, \lambda\left(X_{t}\right)=\lambda X^{2}$, and $\sigma\left(X_{t}\right)=\sigma X_{t}$. To calculate the values for different $M, X, w$ and time horizons, we assume $\theta=0.2, \kappa=0.2, \sigma_{X}=0.15$, $\lambda=0.05$, and $\sigma=0.2$. The unconditional mean is set to $\theta / \kappa=1$. 


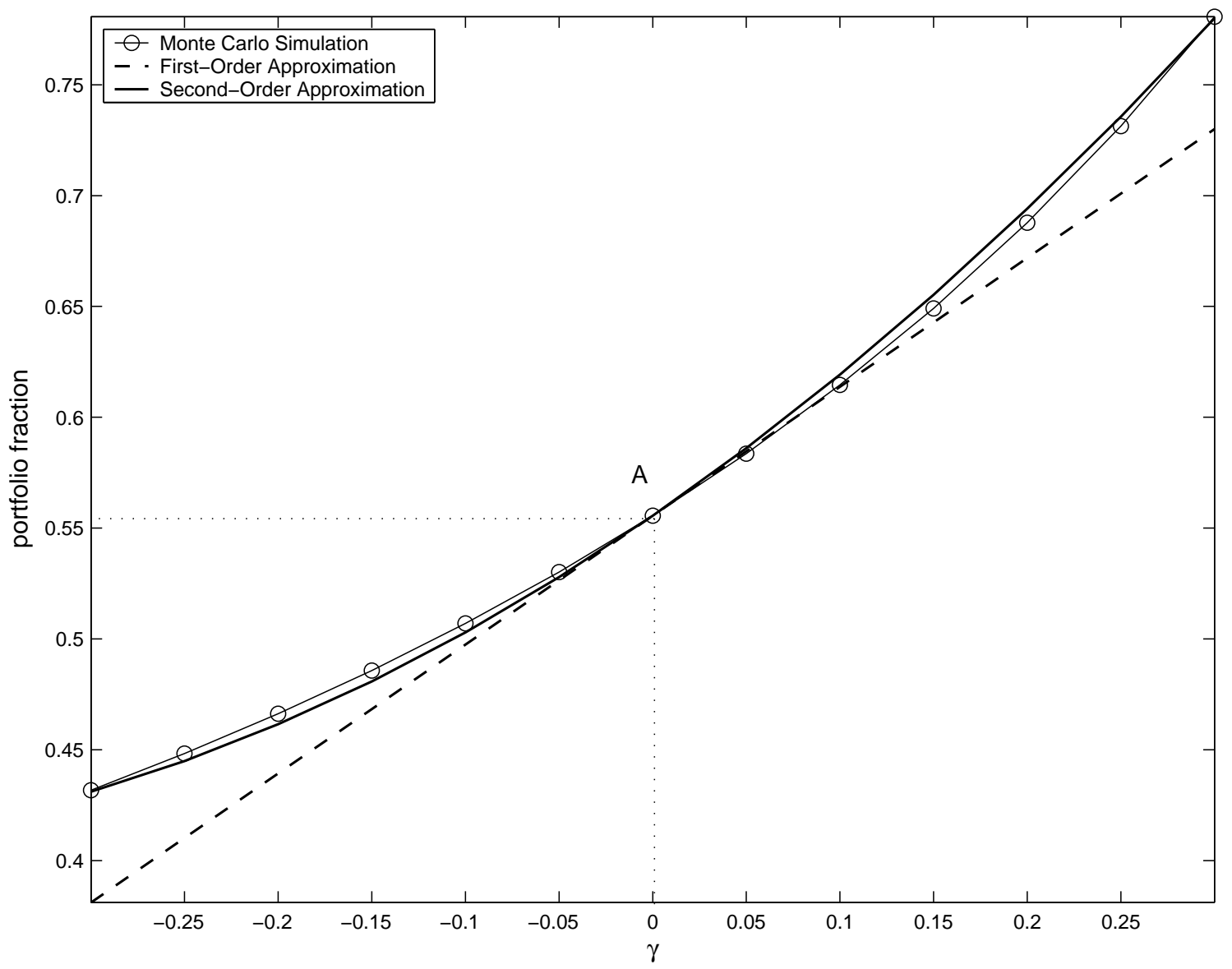

Figure 1: Comparison of the perturbative solution and the exact one computed by Monte Carlo simulation. The model parameters are $\alpha\left(X_{t}\right)=0.05 X_{t}^{2}, \sigma\left(X_{t}\right)=0.25 X_{t}, \theta=\kappa=0.8, \sigma_{X}=$ $0.2, T=1$. For the Monte Carlo solution, we partition the investor's time horizon into 100 time steps. In each time step, we draw $100^{\prime} 000$ random numbers, using antithetic variates. The log investor's policy is given in the middle of the $x$-axis for the value $\gamma=0$. 
(A)

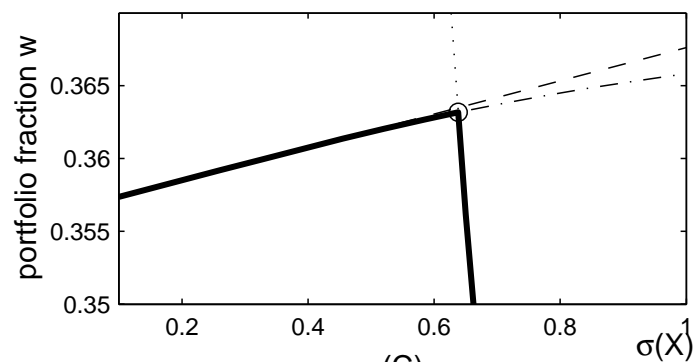

(C)

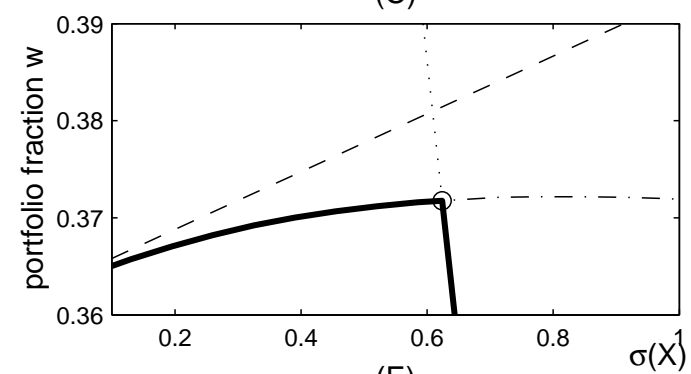

(E)

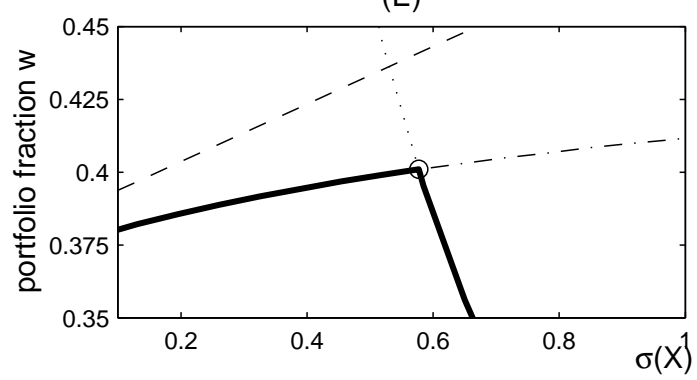

(B)

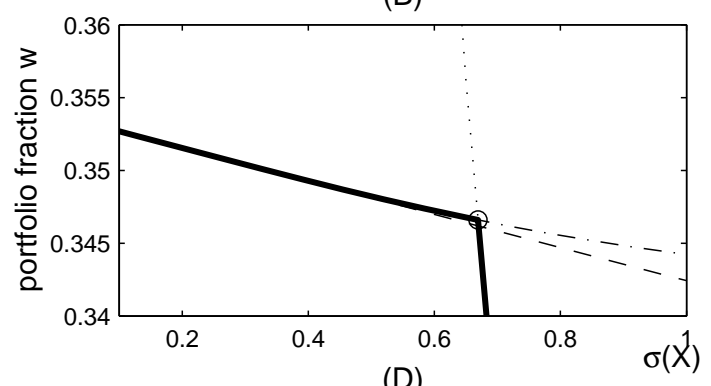

(D)

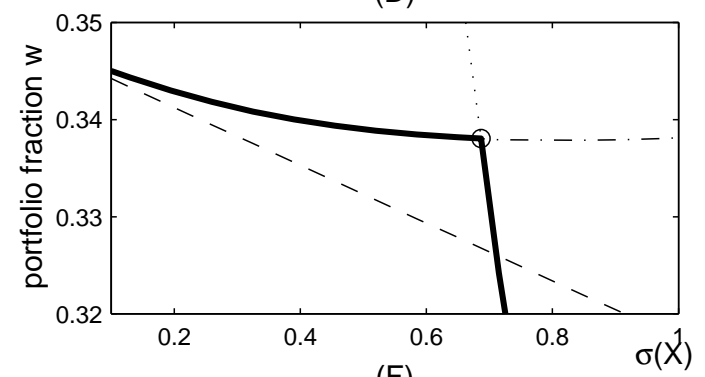

$(\mathrm{F})$

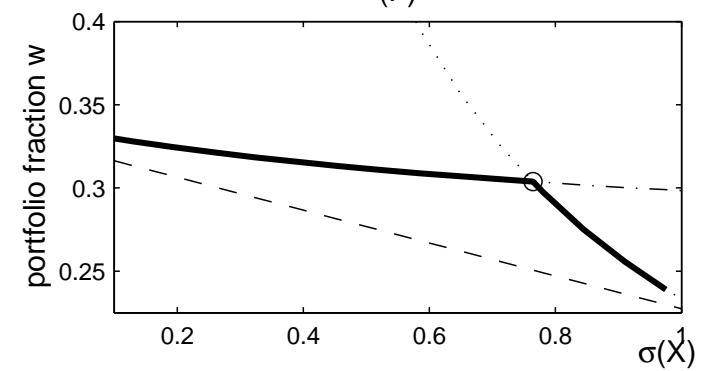

Figure 2: Portfolio policy under VaR regulation and influence of the investment horizon. Graphs on the left assume a positive correlation parameter $\rho=0.4$. Graphs on the right assume $\rho=-0.4$. Graphs (A) and (B) assume an investment horizon of $T=1$ year. Graphs (C) and (D) assume $T=5$ and graphs (E) and (F) assume $T=10$. The solid bold line represents the portfolio policy of the VaR-constrained bank. At the circled point, the bank runs into the VaR constraint represented by the dotted line. The dashed line represents the optimal policy in the absence of constraints. The parameters were chosen as follows: $\gamma=0.5, \nu=0.01, \beta=0.05$, $\tau=10 / 250, r=0.05, \lambda=0.03, \sigma=0.32, \kappa_{X}=\theta_{X}=0.2, \sigma_{X}=0.6$. We perform the calculations using standard Monte Carlo methods. 
(A)
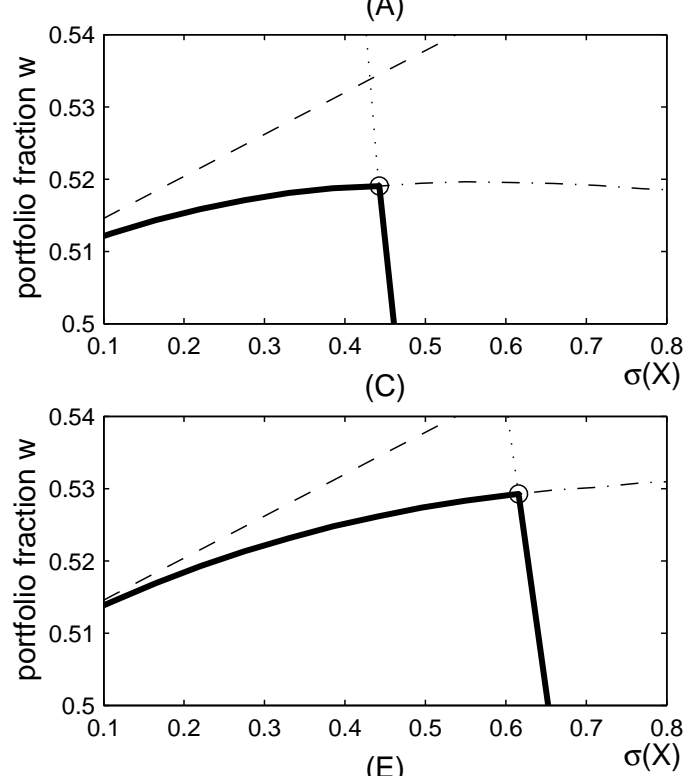

(E)

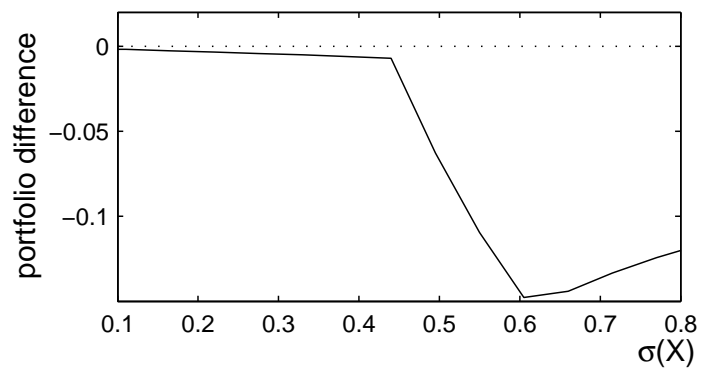

(B)
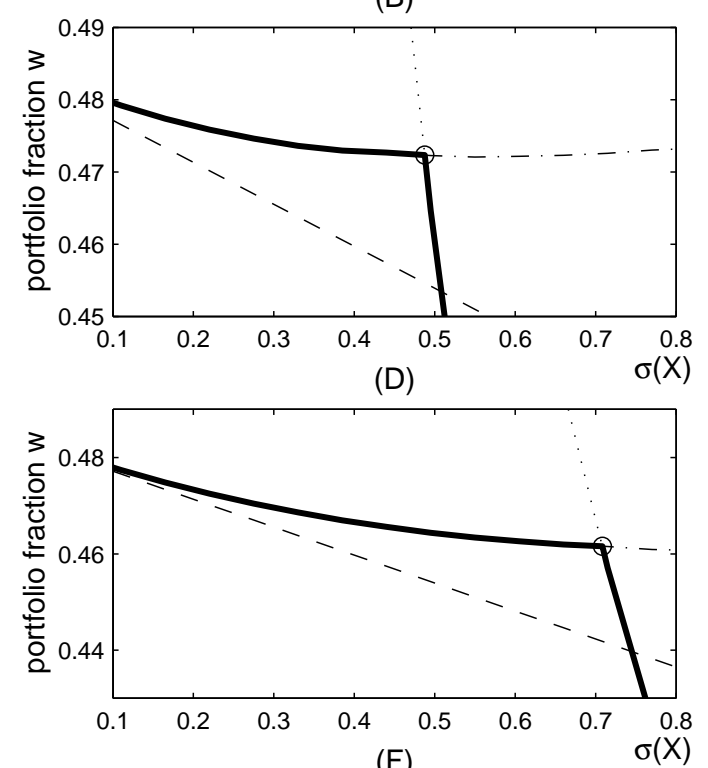

(F)

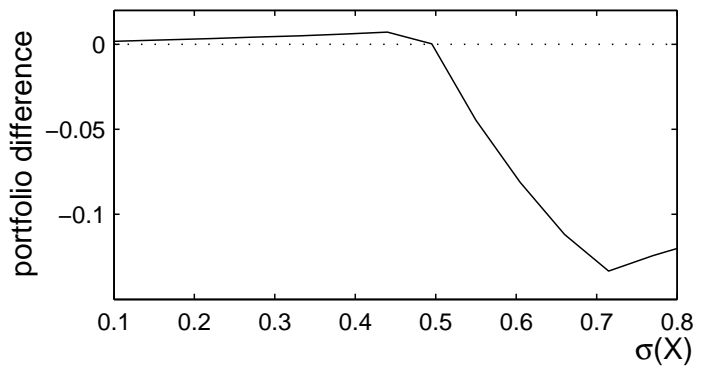

Figure 3: Portfolio policy under VaR regulation and the influence of the confidence level. Graphs on the left side assume $\rho=0.4$. Graphs on the right side assume $\rho=-0.4$. Graphs (A) and (B) assume a confidence bound of $\nu=0.01$. Graphs (C) and (D) assume $\nu=0.05$. Graphs (E) and (D) show the differences in the optimal policies when the confidence level is tightened, i.e. we plot the differences (C)-(A) and (D)-(B). The time horizon is $T=5$ years. The parameters were chosen as follows: $\gamma=0.5, \nu=0.01, \beta=0.05, \tau=10 / 250, r=0.05, \lambda=0.03, \sigma=0.32$, $\kappa_{X}=\theta_{X}=0.2, \sigma_{X}=0.6$. 

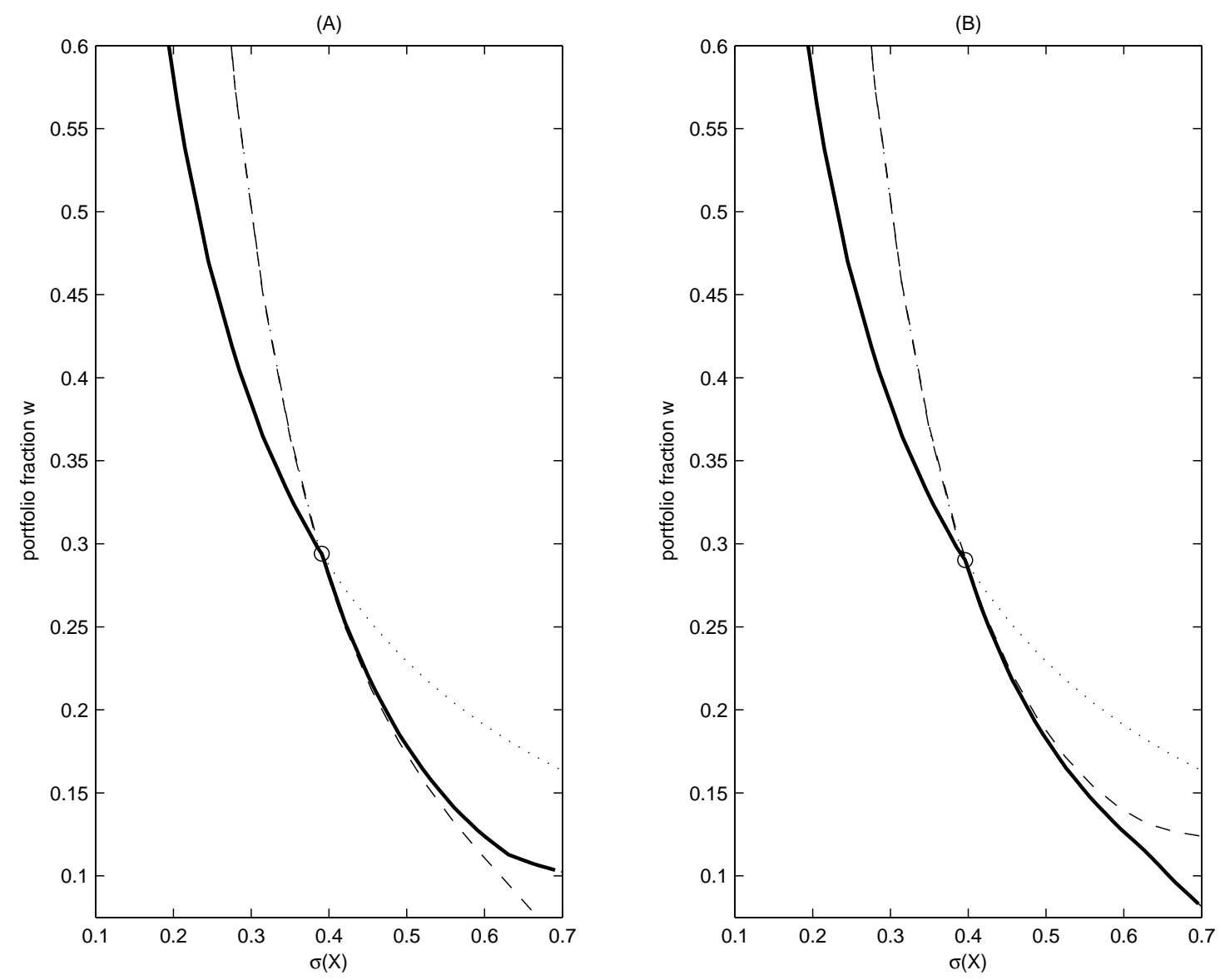

Figure 4: Portfolio policies in a pure volatility model. We plot the portfolio policies when $\lambda\left(X_{t}\right)=\lambda$ and $\sigma\left(X_{t}\right)=\sigma X_{t}$. The dashed line represents the optimal policy of an unconstrained investor. The bold straight line is the portfolio policy of the constrained investor. Panels (A) and (B) assume a positive and a negative correlation parameter $\rho=0.4$ and $\rho=-0.4$, respectively. Further parameters are calibrated as $\gamma=0.5, \nu=0.01, \beta=0.05, \tau=10 / 250, r=0.05$, $\lambda=0.03, \theta=\kappa=0.7, \sigma_{X}=0.8, \sigma=0.35, T=5$. 

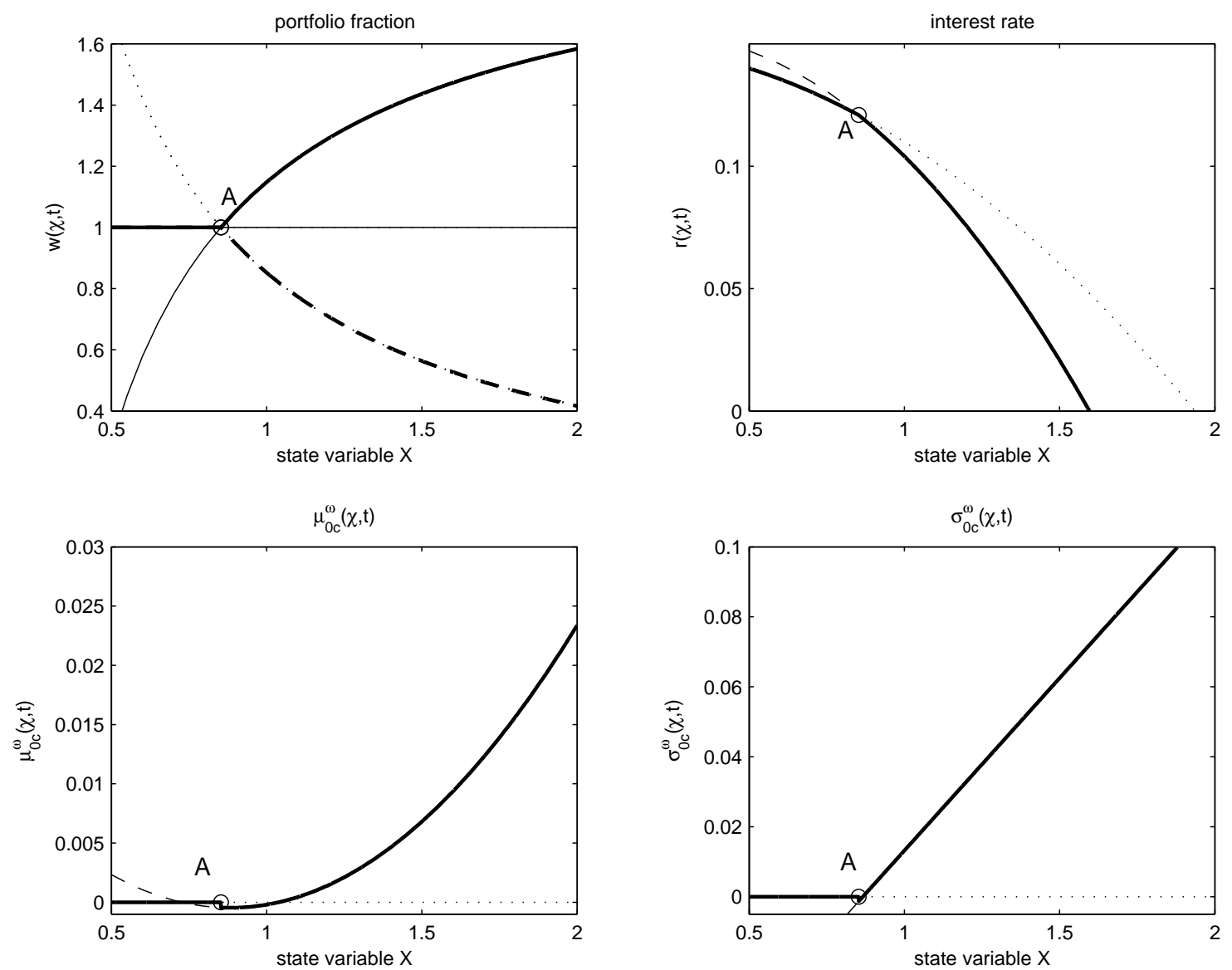

Figure 5: Impact of VaR regulation in the log-economy $M_{0}$. We assume the following parameter values: $\sigma_{e}=0.2, \mu_{e}=0.1, \sigma_{X}=1, \theta=\kappa=0.1, \delta=0.05, T=5, \tau=1 / 250, \beta=2.5 \%, v=$ $5 \%, \rho_{e X}=0.4, \gamma_{I}=\gamma_{I I}=0$. Bold lines represent quantities resulting from the constrained economy. In the panels for the interest rate, the drift, and the volatility of the cross-sectional wealth dynamics, the dotted lines to the right of point $A$ represent quantities in the unconstrained economy. 

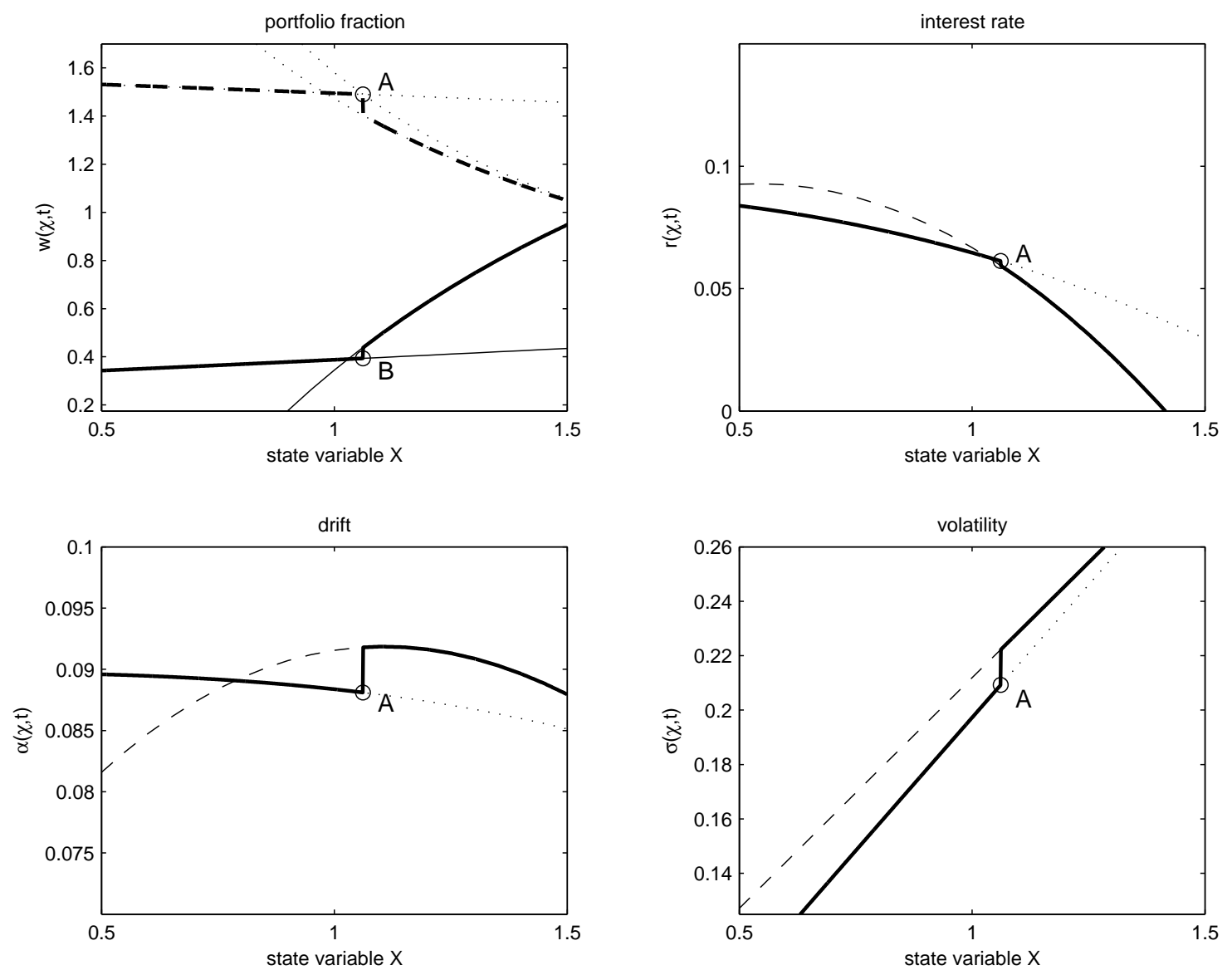

Figure 6: Impact of VaR regulation in model $M_{1}$ with a positive correlation $\rho_{e X}$. We assume $d e_{t} / e_{t}=\mu_{e} d t+\sigma_{e} X_{t} d Z_{t}^{e}$ and the following parameter values: $\sigma_{e}=0.2, \mu_{e}=0.1, \sigma_{X}=1, \theta=$ $\kappa=0.1, \delta=0.1, T=2, \tau=1 / 250, \beta=5 \%, v=1 \%, \rho_{e X}=0.4, \gamma_{I}=-0.1, \gamma_{I I}=0.4$. Bold lines represent quantities resulting from the constrained economy. In the panels for the interest rate, the drift, and the volatility, the dotted lines to the right of point $A$ represent quantities in the unconstrained economy. 


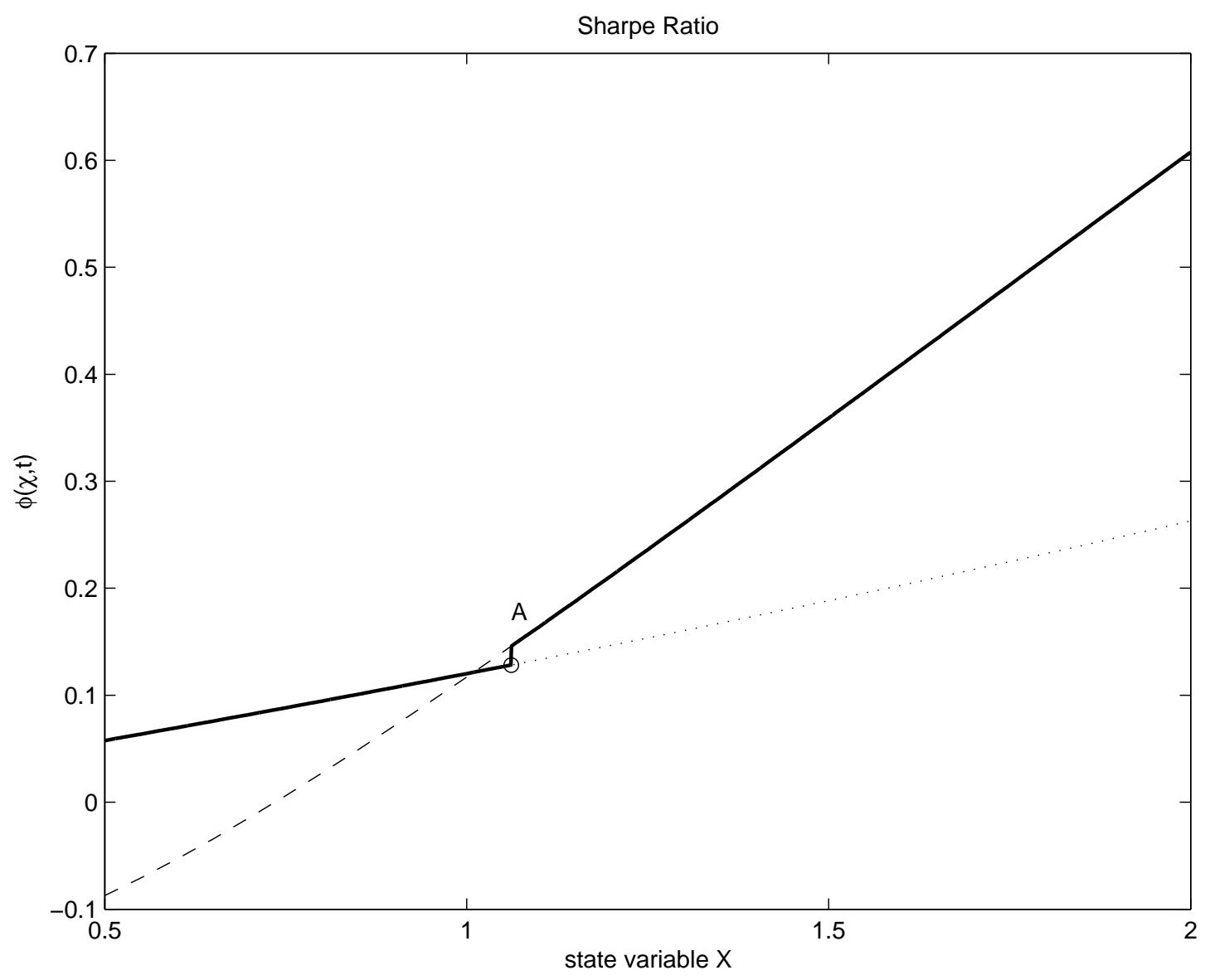

Figure 7: Impact of VaR regulation on Sharpe ratios for model $M_{1}$ with a positive correlation $\rho_{e X}$. We assume $d e_{t} / e_{t}=\mu_{e} d t+\sigma_{e} X_{t} d Z_{t}^{e}$ and the following parameter values: $\sigma_{e}=0.2, \mu_{e}=$ $0.1, \sigma_{X}=1, \theta=\kappa=0.1, \delta=0.1, T=2, \tau=1 / 250, \beta=5 \%, v=1 \%, \rho_{e X}=0.4, \gamma_{I}=-0.1, \gamma_{I I}=$ 0.4. The bold line is the Sharpe ratio resulting from the constrained economy. The dotted line to the right of point $A$ represents the Sharpe ratio in the unconstrained economy. 

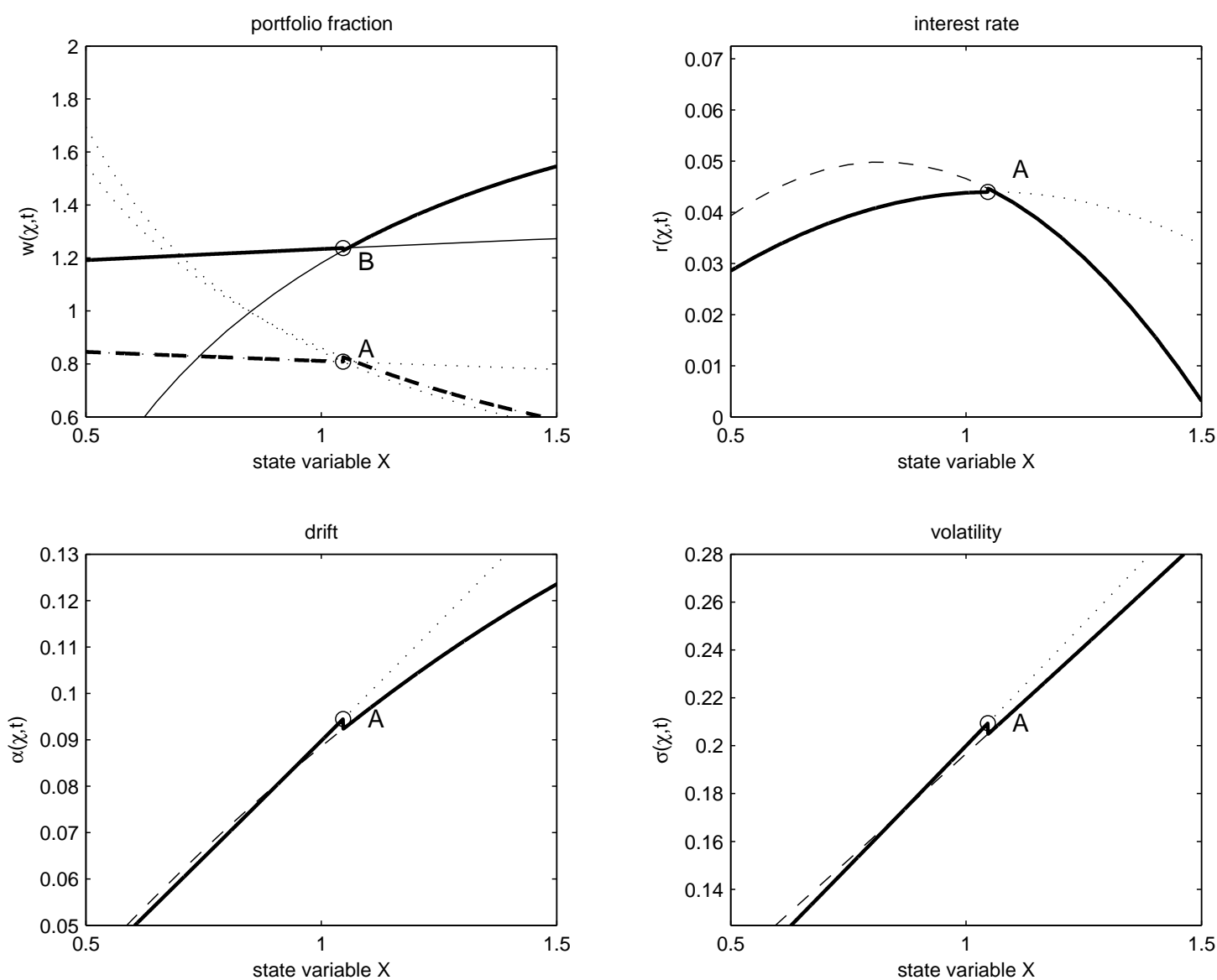

Figure 8: Impact of VaR regulation for model $M_{2}$ with a negative correlation $\rho_{e X}$. We assume $d e_{t} / e_{t}=\mu_{e} X_{t} d t+\sigma_{e} X_{t} d Z_{t}^{e}$ and the following parameter values: $\sigma_{e}=0.2, \mu_{e}=0.1, \sigma_{X}=1, \theta=$ $\kappa=0.1, \delta=0.1, T=2, \tau=1 / 250, \beta=2.5 \%, v=1 \%, \rho_{e X}=-0.4, \gamma_{I}=-0.1, \gamma_{I I}=0.4$. Bold lines represent quantities resulting from the constrained economy. In the panels for the interest rate, the drift, and the volatility of the asset price process, the dotted lines to the right of point $A$ represent the corresponding quantities in the unconstrained economy. 


\section{Appendix A}

Time is discrete and the length between time steps is denoted by $\Delta . \psi_{n}$ denotes the absolute number of stocks $S$ of a portfolio $V$ at time $n$. Then, the change in the relative portfolio fraction $w$ invested in the risky asset $S$ is given by

$$
\begin{aligned}
\Delta w & :=w_{n+1}-w_{n}=\psi_{n+1} \frac{S_{n}+\Delta S}{V_{n}+\Delta V}-\psi_{n} \frac{S_{n}}{V_{n}} \\
& =\psi_{n} \frac{S_{n}+\Delta S}{V_{n}+\Delta V}-\psi_{n} \frac{S_{n}}{V_{n}}+\Delta \psi \frac{S_{n}+\Delta S}{V_{n}+\Delta V} .
\end{aligned}
$$

Using Neumann Series and cancelling out higher-order terms, we get

$$
\begin{aligned}
\Delta w & =\psi_{n} \frac{S_{n}+\Delta S}{V_{n}}\left(1-\frac{\Delta V}{V_{n}}\right)-\psi_{n} \frac{S_{n}}{V_{n}}+\Delta \psi \frac{S_{n}+\Delta S}{V_{n}+\Delta V} \\
& =\psi_{n} \frac{S_{n}}{V_{n}}\left(1-\frac{\Delta V}{V_{n}}\right)+\psi_{n} \frac{\Delta S_{n}}{V_{n}}-\psi_{n} \frac{S_{n}}{V_{n}}+\Delta \psi \frac{S_{n}+\Delta S}{V_{n}+\Delta V} \\
& =-\psi_{n} \frac{S_{n}}{V_{n}} \frac{\Delta V}{V_{n}}+\psi_{n} \frac{\Delta S}{V_{n}}+\Delta \psi \frac{S_{n}+\Delta S}{V_{n}+\Delta V} .
\end{aligned}
$$

When we assume a portfolio strategy with constant absolute values invested in the risky asset, i.e., $\Delta \psi=0$, then

$$
\left.\Delta w\right|_{\Delta \psi=0}=-\psi_{n} \frac{S_{n}}{V_{n}} \frac{\Delta V}{V_{n}}+\psi_{n} \frac{\Delta S}{V_{n}}=\psi_{n} \Delta\left(\frac{S_{n}}{V_{n}}\right) .
$$

To calculate regulatory VaR figures, it is common practice (see e.g. the Riskmetrics document) that VaR is calculated over a one-day holding period and scaled up by the appropriate scaling factor to obtain a ten day VaR. To this end, the drift of the portfolio dynamics is set equal to zero. In this case,

$$
\left.\Delta w\right|_{\Delta \psi=0} \approx 0
$$

\section{Appendix B}

Assumption 2 states that $X_{t}$ follows a mean reverting process given by

$$
d X_{t}=\left(\theta-\kappa X_{t}\right) d t+\sigma_{X} X_{t}^{m} d Z_{t}^{X}
$$


where $\kappa, \theta, \sigma_{X}, m$ are positive constants. The risk premium, the risky asset volatility and the interest rate processes are of the form $\lambda\left(X_{t}\right)=\lambda X_{t}^{n_{1}}, \sigma\left(X_{t}\right)=\sigma X_{t}^{n_{2}}$, and $r\left(X_{t}\right)=r$, where $\lambda, n_{1}, n_{2}, r$ are non-negative constants.

Assumption 2 comprises model settings in which volatility is stochastic or variance-in-mean effects are present, for instance when $n_{1} \neq 0, n_{2} \neq 0$ and $m \neq 0$. On the other hand, when $n_{1}=0$ we can investigate the impact of VaR constraints under a pure stochastic volatility setting. In the case in which $n_{1}=2 n_{2}$, the unconstrained Merton policy of a log-utility investor is a constant $\lambda\left(X_{t}\right) / \sigma\left(X_{t}\right)^{2}=\lambda / \sigma^{2}$.

Given Assumption 2, we can study the functional form of the portfolio bounds $w_{b}^{ \pm}\left(X_{t}\right)$ more explicitly. Using the definition of the VaR constraint in equation (10), it follows from the Implicit Function Theorem and Assumption 2 that

$$
\frac{\partial w_{b}^{ \pm}}{\partial X}=\frac{w_{b}^{ \pm}}{X} \cdot \frac{\left(n_{1}-n_{2}\right) \lambda X^{n_{1}} \tau-n_{2} D}{D}
$$

where

$$
D= \pm \sqrt{\left(\lambda X^{n_{1}} \tau+\sigma X^{n_{2}} \sqrt{\tau} v\right)^{2}+2 \sigma^{2} X^{2 n_{2}} \tau(r \tau-\log (1-\beta))}
$$

Since, as noted above, $w^{+}>0$ and $w^{-}<0$, the sign of (B.2) is determined by the sign of

$$
\left(n_{1}-n_{2}\right) \lambda X^{n_{1}} \tau-n_{2} D
$$

For an explicit model setting, we can now discuss the impact of a change in volatility on the tightness of the VaR constraints. We observe that

$$
\frac{\partial w_{b}^{ \pm}}{\partial X}<0, \text { if } n_{1} \leq n_{2}
$$

Therefore, we see that a higher volatility state $X$ implies lower absolute values of $w_{b}^{ \pm}$and thus a tighter VaR constraint for both long and short portfolio exposures. For more general choices of $n_{1}$ and $n_{2}$ no such absolute statement about the sign of $\frac{\partial w_{b}^{ \pm}}{\partial X}$ can be made for the whole support of $X$. However, the derivative in (B.5) is generally negative for reasonable values of $X$. For example, this is the case when $n_{1}=2, n_{2}=1$. In Section 3.4, this particular choice is discussed in more detail. Restricting the parameter $m$ to $m=\frac{1}{2}$ or $m=1$ we obtain a model class that encompasses the most popular stochastic volatility models, like the lognormal, the square-Gaussian, or the square-root model of Heston (1993). 


\section{Appendix C}

The proof of Proposition 5 implies that the function $g_{00}(\chi, t)$ take the form

$$
g_{00}(\chi, t)=\frac{1}{A_{t}} e^{-\delta(T-t)} \mathbb{E}\left[H_{t, T} \mid \mathcal{F}_{t}\right]+\frac{\int_{t}^{T} e^{-\delta(s-t)}}{A_{t}}\left(\mathbb{E}\left[H_{t, s} \mid \mathcal{F}_{t}\right]+\mathbb{E}\left[\log c_{s} \mid \mathcal{F}_{t}\right]\right) d s
$$

with

$$
\mathbb{E}\left[H_{t, T} \mid \mathcal{F}_{t}\right]=\mathbb{E}\left[\int_{t}^{T}\left(r_{\log }+w_{\log }\left(\alpha_{\log }-r_{\log }\right)-c_{\log }(s)-\frac{1}{2} w_{\log }^{2} \sigma_{\log }^{2}\right) d s \mid \mathcal{F}_{t}\right] .
$$

Because $g_{00}(\chi, t)$ is determined in the homogeneous log-economy, equation (C.2) can be simplified considerably. First, we note that in the log-economy, market clearing implies $w_{\log }=1$. Further, $c_{\log }=1 / A_{t}$ and, as $\gamma_{I}=\gamma_{I I}=0, \alpha_{\log }=\delta+\mu_{e}(X, t), \sigma_{\log }=\sigma_{e}\left(X_{t}\right)$. Inserting the above expressions in $g_{00}(\chi, t)$, and with an endowment and state variable process (41) and (42) it follows

$$
\begin{aligned}
g_{00}(X, t) & =g_{00}^{f}(\chi, t)=g_{00}^{c}(\chi, t) \\
& =\frac{e^{-\delta(T-t)}}{A_{t}}\left(\log A_{t}+\mathbb{E}\left[H_{t, T}^{e} \mid \mathcal{F}_{t}\right]\right)+\frac{1}{A_{t}} \int_{t}^{T} e^{-\delta(s-t)} \mathbb{E}\left[H_{t, s}^{e} \mid \mathcal{F}_{t}\right] d s,
\end{aligned}
$$

where

$$
\mathbb{E}\left[H_{t, T}^{e} \mid \mathcal{F}_{t}\right]=\int_{t}^{T} \mathbb{E}\left[\mu_{e}(X, s)-\frac{1}{2} \sigma_{e}(X, s)^{2} \mid \mathcal{F}_{t}\right] d s
$$

\section{Appendix D}

\section{D.1 Proof of Proposition 1}

With portfolio weights fixed at time $t=0$, the wealth dynamics under Assumption 2 read

$$
\log \mathcal{W}_{t}=\log W_{0}+\int_{0}^{t}\left(r\left(X_{s}\right)+w_{0} \lambda\left(X_{s}\right)-\frac{1}{2} w_{0}^{2} \sigma^{2}\left(X_{s}\right)\right) d s+w_{0} \int_{0}^{t} \sigma\left(X_{s}\right) d Z_{s}
$$

Performing an Itô-Taylor expansion in $X$, we obtain $\log \mathcal{W}=\log \mathcal{W}^{(1)}+\mathbf{R}$ with remainder

$$
\begin{aligned}
\mathbf{R}= & w_{0} \sigma\left(X_{0}\right) \int_{0}^{t} \int_{0}^{s} d Z_{u} d s+\int_{0}^{t} \int_{0}^{s}\left(\mathcal{L}_{0} h_{1}\left(X_{u}\right) d u+\mathcal{L}_{1} h_{1}\left(X_{u}\right) d Z_{u}^{X}\right) d s \\
& +w_{0} \int_{0}^{t} \int_{0}^{s}\left(\mathcal{L}_{0} h_{2}\left(X_{u}\right) d u+\mathcal{L}_{1} h_{2}\left(X_{u}\right) d Z_{u}^{X}\right) d Z_{s},
\end{aligned}
$$


where

$$
\begin{aligned}
h_{1}(X) & =r(X)+w_{0} \lambda(X)+\frac{1}{2} w_{0}^{2} \sigma(X)^{2}, \\
h_{2}(X) & =\sigma(X) \\
\mathcal{L}_{0} & =\mu_{X}(X) \frac{\partial}{\partial X}+\frac{1}{2} \sigma_{X}^{2}(X) \frac{\partial^{2}}{\partial X^{2}} \\
\mathcal{L}_{1} & =\sigma_{X}(X) \frac{\partial}{\partial X} .
\end{aligned}
$$

Using Markov's inequality we get the bound

$$
\mathbb{P}\left(\left|\log \mathcal{W}_{t}^{(1)}-\log \mathcal{W}_{t}\right| \geq M\right) \leq \frac{1}{M} \mathbb{E}\left[\left|\log \mathcal{W}_{t}^{(1)}-\log \mathcal{W}_{t}\right|\right]=\frac{1}{M} \mathbb{E}[|\mathbf{R}|]
$$

Since the first moment of a multiple Itô integral vanishes, if it has at least one integration for a component of the Wiener process, $\mathbb{E}[|\mathbf{R}|]$ follows as claimed.

\section{D.2 Proof of Proposition 2}

From Proposition 1,

$$
\begin{aligned}
\mathbb{P} & \left(\mathcal{W}_{t+\tau}^{(1)}-W_{t} \leq-L \mid \mathcal{F}_{t}\right) \\
& =\mathbb{P}\left(Z_{t+\tau}-Z_{t} \leq \frac{\log (1-\beta)-\left(r\left(X_{t}\right)+w_{t} \lambda\left(X_{t}\right)+\frac{1}{2} w_{t}^{2} \sigma\left(X_{t}\right)^{2}\right) \tau}{w_{t} \sigma\left(X_{t}\right)} \mid \mathcal{F}_{t}\right) \\
& =N\left(\frac{\log (1-\beta)-\left(r\left(X_{t}\right)+w_{t} \lambda\left(X_{t}\right)+\frac{1}{2} w_{t}^{2} \sigma\left(X_{t}\right)^{2}\right) \tau}{w_{t} \sigma\left(X_{t}\right) \sqrt{\tau}}\right)
\end{aligned}
$$

where $\beta=L / W_{t}$ and $N(\cdot)$ is the cumulative normal distribution function. The approximated VaR at the confidence level $\nu$ then reads

$$
\widehat{\operatorname{VaR}}_{t}^{\nu, w}=W_{t}\left(1-e^{\left(r\left(X_{t}\right)+w_{t} \lambda\left(X_{t}\right)-\frac{1}{2} w_{t}^{2} \sigma\left(X_{t}\right)^{2}\right) \tau+v w_{t} \sigma\left(X_{t}\right) \sqrt{\tau}}\right)
$$

which is equivalent to

$$
\log (1-\beta)-\left(r\left(X_{t}\right)+w_{t} \lambda\left(X_{t}\right)-\frac{1}{2} w_{t}^{2} \sigma\left(X_{t}\right)^{2}\right) \tau-v w_{t} \sigma\left(X_{t}\right) \sqrt{\tau} \leq 0
$$

This inequality is equivalent to the upper and lower bound $w_{b}^{ \pm}$stated in the proposition. 


\section{D.3 Proof of Proposition 3}

The Hamilton-Jacobi-Bellman (HJB) equation for the control problem (P1) is

$$
\begin{aligned}
0= & \max _{w}\left\{J_{t}+(\theta-\kappa X) J_{X}+\frac{1}{2} X^{2} \sigma_{X}^{2} J_{X X}+W\left(r+w \mu X^{2}\right) J_{W}\right. \\
& +w W X^{2} \rho \sigma \sigma_{X} J_{W X}+\frac{1}{2} w^{2} W^{2} \sigma^{2} X^{2} J_{W W} \\
& \left.-\phi\left(\log (1-\beta)-\left(r+w \mu X^{2}-\frac{1}{2} w^{2} \sigma^{2} X^{2}\right) \tau-w \sigma \sqrt{\tau} X N^{-1}(\nu)\right)\right\} \\
=: & \max _{w}(-\phi Q(w)+\mathcal{G} J)
\end{aligned}
$$

with the terminal condition $J(W, X, T)=u(W, X)$. The first-order conditions for the problem are

$$
\begin{aligned}
0 & =\frac{\partial}{\partial w}(-\phi Q(w)+\mathcal{G} J) \\
0 & =\phi Q(w) \\
0 & \geq Q(w)
\end{aligned}
$$

From the first-order condition, we formally get

$$
\begin{aligned}
& w\left(W^{2} J_{W W}-\tau \phi X^{2}\right)= \\
& -\frac{1}{\sigma^{2} X^{2}}\left(\mu X^{2} \tau \phi+\sigma X \sqrt{\tau} \phi N^{-1}(\nu)+W \mu J_{W}+W X^{2} \rho \sigma \sigma_{X} J_{W X}\right)
\end{aligned}
$$

Since the terms in brackets are functions of $W, X$, and $t$ there exists a function $\phi$ that satisfies conditions (D.13)-(D.15). The inequality (D.15) is equivalent to $w_{b}^{-} \leq w \leq w_{b}^{+}$as in Proposition 2. If the VaR constraint does not bind, slackness implies

$$
w_{f}=\frac{W \mu J_{W}+W \rho \sigma \sigma_{X} J_{W X}}{W^{2} \sigma^{2} J_{W W}} .
$$

If $w_{f}(X, t) \geq w_{b}^{+}(X, t), J$ solves

$$
\begin{aligned}
0 & =J_{t}+(\theta-\kappa X) J_{X}+\frac{1}{2} \sigma_{X}^{2} X^{2} J_{X X} \\
& +\left(r+\mu X^{2} w^{+}\right) W J_{W}+\rho w^{+} \sigma \sigma_{X} X^{2} W X J_{W X}+\frac{1}{2} \sigma^{2} X^{2}\left(w^{+}\right)^{2} W^{2} J_{W W}
\end{aligned}
$$

and the same PDE holds if $w_{f} \leq w_{b}^{-}(X, t)$ with $w_{b}^{+}(X, t)$ replaced by $w_{b}^{-}(X, t)$. 


\section{D.4 Proof of Proposition 4}

We calculate the value function for a log-investor as

$$
\begin{aligned}
J_{\log }(W, X, t) & =\mathbb{E}\left[\log W_{T} \mid \mathcal{F}_{t}\right] \\
& =\log W_{t}+\int_{t}^{T}\left(r+\mathbb{E}\left[w_{\log } \lambda\left(X_{s}\right) \mid \mathcal{F}_{t}\right]-\frac{1}{2} \mathbb{E}\left[w_{\log }^{2} \sigma^{2}\left(X_{s}\right) \mid \mathcal{F}_{t}\right]\right) d s,
\end{aligned}
$$

with

$$
w_{\log }=\left\{\begin{array}{lll}
w_{\log }^{f} & \text { if } \quad w_{b}^{-}<w_{\log }^{f}<w_{b}^{+}, \\
w_{b}^{+} & \text {if } \quad w_{\log }^{f} \geq w_{b}^{+}, \\
w_{b}^{-} & \text {if } \quad w_{\log }^{f} \leq w_{b}^{-},
\end{array}\right.
$$

where $w_{\log }^{f}=X^{n_{1}-2 n_{2}} \frac{\lambda}{\sigma^{2}}$ is the log investor's portfolio policy in the unconstrained case. Since $X>0$, the only constraint binding for the log investor, given Assumption 2, is $w_{b}^{+}$. Since

$$
J_{\log }(W, X, t)=\lim _{\gamma \rightarrow 0} \frac{e^{\gamma\left(g_{0}(X, t)+\gamma g_{1}(X, t)\right)} W_{t}^{\gamma}-1}{\gamma}=\log W_{t}+g_{0}(X, t),
$$

formulas (D.19) and (D.21) for the unconstrained function $g_{0}^{f}(X, t)$ follow directly. Accounting for the presence of constraints, we have

$$
\begin{aligned}
g_{0}(X, t)= & r(T-t)+\lambda \int_{t}^{T} \mathbb{E}\left[w_{\log }^{f}\left(X_{s}\right)+\mathbb{1}_{\left\{\left|w_{\log }^{f}\right|>\left|w_{b}^{ \pm}\right|\right\}} \hat{w}_{b}^{ \pm}\left(X_{s}\right) X_{s}^{n_{1}} \mid \mathcal{F}_{t}\right] d s \\
& -\frac{1}{2} \sigma^{2} \int_{t}^{T} \mathbb{E}\left[\left(w_{\log }^{f}\left(X_{s}\right)+\mathbb{1}_{\left\{\left|w_{\log }^{f}\right|>\left|w_{b}^{ \pm}\right|\right\}} \hat{w}_{b}^{ \pm}\left(X_{s}\right)\right)^{2} X_{s}^{2 n_{2}} \mid \mathcal{F}_{t}\right] d s \\
= & r(T-t)+\lambda \int_{t}^{T} \mathbb{E}\left[w_{\log }^{f}\left(X_{s}\right)+\mathbb{1}_{\left\{\hat{w}_{b}^{+}<0\right\}} \hat{w}_{b}^{+}\left(X_{s}\right) X_{s}^{n_{1}} \mid \mathcal{F}_{t}\right] d s \\
& -\frac{1}{2} \sigma^{2} \int_{t}^{T} \mathbb{E}\left[\left(w_{\log }^{f}\left(X_{s}\right)+\mathbb{1}_{\left\{\hat{w}_{b}^{+}<0\right\}} \hat{w}_{b}^{+}\left(X_{s}\right)\right)^{2} X_{s}^{2 n_{2}} \mid \mathcal{F}_{t}\right] d s \\
= & g_{0}^{f}(X, t)-\frac{1}{2} \int_{t}^{T} \mathbb{E}\left[\mathbb{1}_{\left\{\hat{w}_{b}^{+}<0\right\}}\left(\hat{w}_{b}^{+}\left(X_{s}\right) \sigma X_{s}^{n_{2}}\right)^{2} \mid \mathcal{F}_{t}\right] d s,
\end{aligned}
$$

where $\hat{w}_{b}^{+}=w_{b}^{+}-w_{\log }^{f}$. Since $X_{s}>0$ for all $s \in[t, T]$, we can define $\phi(X):=\hat{w}_{b}^{+} \sigma X^{n_{2}}$ and make the corresponding substitutions in (D.24). Thus, we obtain

$$
\begin{aligned}
g_{0}(X, t) & =g_{0}^{f}(X, t)-\frac{1}{2} \int_{t}^{T} \mathbb{E}\left[\mathbb{1}_{\left\{\phi\left(X_{s}\right)<0\right\}} \phi\left(X_{s}\right)^{2} \mid \mathcal{F}_{t}\right] d s, \\
g_{0}^{f}(X, t) & =r(T-t)+\frac{1}{2} \frac{\lambda^{2}}{\sigma^{2}} \int_{t}^{T} \mathbb{E}\left[X_{s}^{2\left(n_{1}-n_{2}\right)} \mid \mathcal{F}_{t}\right] d s,
\end{aligned}
$$


where

$$
\phi\left(X_{t}\right)=\frac{v}{\sqrt{\tau}}+\frac{\sqrt{\left(\lambda X_{t}^{n_{1}} \tau+\sigma X_{t}^{n_{2}} \sqrt{\tau} v\right)^{2}+2 \sigma^{2} X_{t}^{2 n_{2}} \tau(r \tau-\log (1-\beta))}}{\sigma X_{t}^{n_{2}} \tau} .
$$

and $g_{0}^{f}$ is the " $g_{0}$-function" prevailing in the absence of VaR constraints. $g_{0}^{c}$ immediately follows.

\section{D.5 Proof of Corollary 1}

Plugging the bound $K X^{2 j\left(n_{1}-n_{2}\right)}$ into equation (20), we can use the Euler gamma function $\Gamma(a)$ and the incomplete Gamma function, $\Gamma(b, a)$ to obtain

$$
\begin{aligned}
\left|w_{f}^{(n)}(X, t)\right| \leq & \left|X^{n_{1}-2 n_{2}} \frac{\lambda}{\sigma^{2}} \frac{1-\gamma^{n+1}}{1-\gamma}\right| \\
& +\left|\frac{\rho \sigma_{X}}{\sigma} \gamma K X^{m-n_{2}} \frac{e^{X_{t}^{2\left(n_{1}-n_{2}\right)}} \gamma\left(n, X_{t}^{2\left(n_{1}-n_{2}\right)} \gamma\right)-\gamma^{n} e^{X_{t}^{2\left(n_{1}-n_{2}\right)}} \Gamma\left(n, X_{t}^{2\left(n_{1}-n_{2}\right)}\right)}{(1-\gamma) \Gamma(n)}\right|
\end{aligned}
$$

Since $\lim _{b \rightarrow \infty} \Gamma(b, a) / \Gamma(b)=1$ for finite $a$, the claim follows.

\section{D.6 Proof of Proposition 5}

The HJB equation for the control problem $(\mathbf{P} 2)$ is

$$
\begin{aligned}
0= & \max _{w, c}\left\{J_{t}+\mu_{X}(X) J_{X}+\frac{1}{2} \sigma_{X}(X)^{2} J_{X X}+W(r-c+w \lambda(X)) J_{W}\right. \\
& +w W \rho \sigma(X) \sigma_{X}(X) J_{W X}+\frac{1}{2} w^{2} W^{2} \sigma(X)^{2} J_{W W} \\
& \left.\quad-\phi\left(\log (1-\beta)-\left(r-c+w \lambda(X)-\frac{1}{2} w^{2} \sigma(X)^{2}\right) \tau-w \sigma(X) \sqrt{\tau} v\right)\right\} \\
= & : \max _{w, c}\{\mathcal{G} J-\phi Q(w, c)\} .
\end{aligned}
$$

By the homogeneity properties of the utility function, the solution takes the form

$$
J(W, X, t)=\frac{A_{t}}{\gamma}\left(e^{\gamma g(X, t)} W^{\gamma}-1\right)
$$


The first-order conditions and slackness imply unconstrained optimal policies today, which are given by

$$
\begin{aligned}
w^{f} & =-X^{n_{1}-2 n_{2}} \frac{\lambda J_{W}}{\sigma^{2} W J_{W W}}-\rho X^{m-n_{2}} \frac{\sigma_{X} J_{W X}}{\sigma W J_{W W}} \\
& =X_{t}^{n_{1}-2 n_{2}} \frac{\lambda}{\sigma^{2}(1-\gamma)}+\frac{\gamma}{1-\gamma} X_{t}^{m-n_{2}} \frac{\rho \sigma_{X}}{\sigma} \frac{\partial g(X, t)}{\partial X}, \\
c^{f} & =\left(J_{W} W\right)^{\frac{1}{\gamma-1}}=\left(A_{t} e^{\gamma g(X, t)}\right)^{\frac{1}{\gamma-1}} .
\end{aligned}
$$

We expand $g(X, t)=g_{0}(X, t)+\gamma g_{1}(X, t)+O\left(\gamma^{2}\right)$. From (D.28), the log investor's value function reads

$$
J(W, X, t)=A_{t}\left(\log \left(W_{t}\right)+g_{0}(X, t)\right) .
$$

Expanding the optimal portfolio weight and optimal expenditure rate up to first order, we obtain $w_{f}^{(1)}(X, t)$ and $c_{f}^{(1)}(X, t)$ as claimed. To obtain the function $g_{0}(X, t)$, we recall the wealth dynamics in the presence of intermediate expenditure given in equation (28). A second representation for the value function $J$ is then

$$
\begin{aligned}
J(W, X, t)= & e^{-\delta(T-t)}\left(\log W_{t}+\mathbb{E}\left[H_{t, T} \mid \mathcal{F}_{t}\right]\right) \\
& +\int_{t}^{T} e^{-\delta(s-t)}\left(\log W_{t}+\mathbb{E}\left[H_{t, s} \mid \mathcal{F}_{t}\right]+\mathbb{E}\left[\log c_{s} \mid \mathcal{F}_{t}\right]\right) d s
\end{aligned}
$$

Equating the last two expressions for the value function, it follows that

$$
\begin{aligned}
A_{t} & =e^{-\delta(T-t)}+\frac{1-e^{-\delta(T-t)}}{\delta} \\
g_{0}(X, t) & =\frac{1}{A_{t}} e^{-\delta(T-t)} \mathbb{E}\left[H_{t, T} \mid \mathcal{F}_{t}\right]+\frac{1}{A_{t}} \int_{t}^{T} e^{-\delta(s-t)}\left(\mathbb{E}\left[H_{t, s} \mid \mathcal{F}_{t}\right]+\mathbb{E}\left[\log c_{s} \mid \mathcal{F}_{t}\right]\right) d s .
\end{aligned}
$$

We must still determine the function $H_{t, T}$. Its general form is given in equation (29). As in Proposition 4 , the structure of $w_{b}^{ \pm}(X, t)$ in the presence of intermediate expenditure leads to a decomposition of $H_{t, T}$ as below: ${ }^{5}$

$$
\begin{aligned}
\mathbb{E}\left[H_{t, T} \mid \mathcal{F}_{t}\right] & =\mathbb{E}\left[H_{t, T}^{f} \mid \mathcal{F}_{t}\right]+\mathbb{E}\left[H_{t, T}^{c} \mid \mathcal{F}_{t}\right] \\
\mathbb{E}\left[H_{t, T}^{f} \mid \mathcal{F}_{t}\right] & =r(T-t)-\int_{t}^{T} A_{s}^{-1} d s+\frac{1}{2} \frac{\lambda^{2}}{\sigma^{2}} \int_{t}^{T} \mathbb{E}\left[X_{s}^{2\left(n_{1}-n_{2}\right)} \mid \mathcal{F}_{t}\right] d s, \\
\mathbb{E}\left[H_{t, T}^{c} \mid \mathcal{F}_{t}\right] & =-\frac{1}{2} \int_{t}^{T} \mathbb{E}\left[\mathbb{I}_{\left\{\phi\left(X_{s}, s\right)<0\right\}} \phi\left(X_{s}, s\right)^{2} \mid \mathcal{F}_{t}\right] d s,
\end{aligned}
$$


with

$$
\phi(X, t)=\frac{v}{\sqrt{\tau}}+\frac{\sqrt{\left(\lambda X^{n_{1}} \tau+\sigma X^{n_{2}} \sqrt{\tau} v\right)^{2}+2 \sigma^{2} X^{2 n_{2}} \tau\left(\left(r-A_{t}^{-1}\right) \tau-\log (1-\beta)\right)}}{\sigma X^{n_{2}} \tau} .
$$

Then,

$$
\begin{gathered}
g_{0}^{f}(X, t)=\frac{1}{A_{t}} e^{-\delta(T-t)} \mathbb{E}\left[H_{t, T}^{f} \mid \mathcal{F}_{t}\right]+\frac{1}{A_{t}} \int_{t}^{T} e^{-\delta(s-t)}\left(\mathbb{E}\left[H_{t, s}^{f} \mid \mathcal{F}_{t}\right]-\log A_{s}\right) d s \\
g_{0}^{c}(X, t)=\frac{1}{A_{t}} e^{-\delta(T-t)} \mathbb{E}\left[H_{t, T}^{c} \mid \mathcal{F}_{t}\right]+\frac{1}{A_{t}} \int_{t}^{T} e^{-\delta(s-t)}\left(\mathbb{E}\left[H_{t, s}^{c} \mid \mathcal{F}_{t}\right]-\log A_{s}\right) d s
\end{gathered}
$$

\section{D.7 Proof of Corollary 3}

To prove the impact on expenditure $c_{t}$, we note that from Proposition 5 it follows immediately $g^{f}(X, t)>$ $g_{0}(X, t)$ since $\mathbb{E}\left[H_{t, T}^{f} \mid \mathcal{F}_{t}\right]>\mathbb{E}\left[H_{t, T} \mid \mathcal{F}_{t}\right]$. To prove the impact on the portfolio fractions, we must consider the difference

$$
\frac{\partial g_{0}(X, t)}{\partial X}-\frac{\partial g^{f}(X, t)}{\partial X}
$$

The sign of (D.43) is just the opposite of the derivative of

$$
M\left(X_{t}\right):=\int_{t}^{T} \mathbb{E}\left[\mathbb{1}_{\left\{\phi\left(X_{s}\right)<0\right\}} \phi\left(X_{s}\right)^{2} \mid \mathcal{F}_{t}\right] d s
$$

Then,

$$
\begin{aligned}
\frac{\partial M\left(X_{t}\right)}{\partial X_{t}} & =\int_{t}^{T} \mathbb{E}\left[\frac{\partial X_{s}}{\partial X_{t}} \frac{\partial}{\partial X_{s}}\left(\mathbb{1}_{\left\{\phi\left(X_{s}\right)<0\right\}} \phi\left(X_{s}\right)^{2}\right) \mid \mathcal{F}_{t}\right] d s \\
& =\int_{t}^{T} \mathbb{E}\left[\frac{\partial X_{s}}{\partial X_{t}} \phi\left(X_{s}\right)^{2} \frac{\partial \phi\left(X_{s}\right)}{\partial X_{s}}\left(\delta_{\left\{\phi\left(X_{s}\right)=0\right\}}+\frac{2 \mathbb{1}_{\left\{\phi\left(X_{s}\right)<0\right\}}}{\phi\left(X_{s}\right)}\right) \mid \mathcal{F}_{t}\right] d s \\
& =2 \int_{t}^{T} \mathbb{E}\left[\frac{\partial X_{s}}{\partial X_{t}} \mathbb{1}_{\left\{\phi\left(X_{s}\right)<0\right\}} \phi\left(X_{s}\right) \frac{\partial \phi\left(X_{s}\right)}{\partial X_{s}} \mid \mathcal{F}_{t}\right] d s,
\end{aligned}
$$

where by $\delta_{\{\cdot\}}$ we denote the Dirac-delta function.

For the $X$ 's dynamics considered in the paper, we have $\partial X_{s} / \partial X_{t} \geq 0$ for all $s \geq t$. Moreover, the sign of the expression within the expectation operator depends on the sign of $\frac{\partial X}{\partial X_{t}} \mathbb{1}_{\{\phi(X)<0\}} \phi(X)$. Since $\phi\left(X_{s}\right)$ is multiplied by the index function, it immediately follows $\mathbb{1}_{\left\{\phi\left(X_{s}\right)<0\right\}} \phi\left(X_{s}\right) \leq 0$. Direct computation of 
$\frac{\partial \phi(X)}{\partial X}$ gives

$$
\begin{aligned}
\frac{\partial \phi(X)}{\partial X} & =\frac{\lambda X^{n_{1}-n_{2}-1}}{\sigma D}\left(n_{1}-n_{2}\right)\left(X^{2 n_{2}} w_{b}^{ \pm}(X) \sigma^{2} \tau-D\right) \\
& =\left(n_{1}-n_{2}\right) \frac{\lambda}{\sigma D} X^{n_{1}-n_{2}-1}\left(v \sigma X^{n_{2}} \sqrt{\tau}+\lambda X^{n_{1}} \tau+D\left(\sigma^{2} X^{2 n_{2}} \tau-1\right)\right)
\end{aligned}
$$

with $D$ given in (B.3), which proves the corollary.

\section{D.8 Proof of Proposition 6}

To obtain the equilibrium interest rate $r^{f}(\chi, t)$ we start with the market clearing condition

$$
\omega_{t}^{I}\left(\frac{\alpha(\chi, t)-r(\chi, t)}{\|\sigma(\chi, t)\|^{2}}\left(1+\gamma_{I}\right)+\gamma_{I} \eta_{t}\right)+\left(1-\omega_{t}^{I}\right)\left(\frac{\alpha(\chi, t)-r(\chi, t)}{\|\sigma(\chi, t)\|^{2}}\left(1+\gamma_{I I}\right)+\gamma_{I I} \eta_{t}\right)=1
$$

Solving for $r(\chi, t)$ and expanding in $\gamma$ gives the equilibrium interest rate. To determine $d \omega_{t}^{I}$, we recall that $\omega_{t}^{I}=W_{t}^{I} / P_{t}$. Applying Itô's Lemma to cross-sectional wealth, by inserting the wealth differentials and dropping the $O\left(\gamma^{2}\right)$ terms, we obtain the expression for $d \omega_{t}^{I f}$ up to second order in $\gamma$. To derive the price process, we consider

$$
\frac{d P_{t}}{P_{t}}=\frac{d\left(P_{t} / e_{t}\right)}{P_{t} / e_{t}}+\frac{d e_{t}}{e_{t}}+\frac{d e_{t}}{e_{t}} \frac{d\left(P_{t} / e_{t}\right)}{P_{t} / e_{t}},
$$

with the ratio $P_{t} / e_{t}$ given by

$$
\frac{P_{t}}{e_{t}}=\frac{1}{\omega_{t}^{I}\left(c_{t}^{I}-c_{t}^{I I}\right)+c_{t}^{I I}}=A_{t}\left(1+A_{t} \xi_{t} \Delta\right)+O\left(\gamma^{2}\right) .
$$

Then,

$$
\begin{aligned}
\frac{d P_{t}}{P_{t}}= & \frac{d e_{t}}{e_{t}}+\left(\partial_{t} \log A_{t}+\xi_{t} \Delta \partial_{t} A_{t}\right) d t+\left(\gamma_{I}-\gamma_{I I}\right) A_{t} \xi_{t}\left(d \omega_{t}^{I}+\sigma_{e}\left(X_{t}\right) d\left\langle Z^{e}, \omega^{I}\right\rangle_{t}\right) \\
& +A_{t} \Delta\left(d \xi_{t}+\sigma_{e}\left(X_{t}\right) d\left\langle Z^{e}, \xi\right\rangle_{t}\right)+\left(\gamma_{I}-\gamma_{I I}\right) A_{t} d\left\langle\omega^{I}, \xi\right\rangle_{t}+O\left(\gamma^{2}\right) .
\end{aligned}
$$


Since the bracket processes can be written as

$$
\begin{aligned}
d\left\langle Z^{e}, \omega^{I}\right\rangle_{t} & =\rho_{e X} \sigma_{X}\left(X_{t}\right) \frac{\partial \omega_{t}^{I}}{\partial X_{t}} d t+e_{t} \sigma_{e}\left(X_{t}\right) \frac{\partial \omega_{t}^{I}}{\partial e_{t}} d t \\
d\left\langle Z^{e}, \xi\right\rangle_{t} & =\rho_{e X} \sigma_{X}\left(X_{t}\right) \frac{\partial \xi_{t}}{\partial X_{t}} d t \\
d\left\langle\omega^{I}, \xi\right\rangle_{t} & =e_{t} \rho_{e X} \sigma_{e}\left(X_{t}\right) \sigma_{X}\left(X_{t}\right) \frac{\partial \xi_{t}}{\partial X_{t}} \frac{\partial \omega_{t}^{I}}{\partial e_{t}} d t+\sigma_{X}\left(X_{t}\right)^{2} \frac{\partial \xi_{t}}{\partial X_{t}} \frac{\partial \omega_{t}^{I}}{\partial X_{t}} d t
\end{aligned}
$$

we insert the above expressions into (D.53) and (D.51). Since in an unconstrained homogeneous loginvestor economy $d \omega_{t}^{I}=O(\gamma) d t+O(\gamma) d Z_{t}^{X}$, both the instantaneous drift and volatility of the cumulative price process, $\left(d P_{t}+e_{t} d t\right) / P_{t}$, follow as claimed.

\section{D.9 Proof of Proposition 7}

We assume without loss of generality that $\gamma_{I}<\gamma_{I I}$ and that the constraint for the less risk-averse investor II is binding. Market clearing implies

$$
\omega_{t}^{I}\left(\frac{\alpha^{c}(\chi, t)-r^{c}(\chi, t)}{\left\|\sigma^{c}(\chi, t)\right\|^{2}}\left(1+\gamma_{I}\right)+\gamma_{I} \eta_{t}^{c}\right)+\left(1-\omega_{t}^{I}\right) w_{b}^{+}(\chi, t)=1
$$

Solving for the interest rate and expanding in $\tau$ and $\gamma$, we obtain the interest rate $r\left(\chi^{c}, t\right)$ as given in the proposition with

$$
B=\omega_{t}^{I} v^{2}+2 \log (1-\beta)\left(\omega_{t}^{I}-2\right), \quad h\left(\gamma_{I} ; \tau\right)=\frac{1}{\sqrt{\tau}} \varrho_{\tau, 0}+\sqrt{\tau} \varrho_{\tau, 1}+\tau \varrho_{\tau, 2}+\gamma_{I} \varrho_{\gamma}
$$

where

$$
\begin{aligned}
\varrho_{\tau, 0}= & \left\|\sigma^{c}(\chi, t)\right\| \frac{1-\omega_{t}^{I}}{2-\omega_{t}^{I}}\left(v+\sqrt{B} \omega_{t}^{I}\right) \\
\varrho_{\tau, 1}= & -\frac{\left(1-\omega_{t}^{I}\right) \omega_{t}^{I}\left\|\sigma^{c}(\chi, t)\right\|\left(2 B\left(\alpha^{c}\left(\chi^{c}, t\right)-\frac{1}{A_{t}}-\frac{\left\|\sigma^{c}(\chi, t)\right\|^{2}}{2}\right)+v^{2}\left\|\sigma^{c}(\chi, t)\right\|^{2}\left(\omega_{t}^{I}-2\right)\right)}{2\left(B \omega_{t}^{I c}\right)^{3 / 2}},(\mathrm{D}) \\
\varrho_{\tau, 2}= & \frac{v\left\|\sigma^{c}(\chi, t)\right\|\left(\omega_{t}^{I}-2\right)}{B} \varrho_{\tau, 1} \\
\varrho_{\gamma}= & -\frac{\left\|\sigma^{c}(\chi, t)\right\|^{2}\left(1+\eta_{t}^{c}\right)}{\omega_{t}^{I}-2} \\
& -\frac{\left.\omega_{t}^{I}\left(1-\omega_{t}^{I}\right) v\left\|\sigma^{c}(\chi, t)\right\|^{2}\left(2 \log (1-\beta)\left(\omega_{t}^{I}-2\right)+\left(B\left(1+\eta_{t}^{c}\right)-v^{2}\left(\omega_{t}^{I}-2\right)\right) \omega_{t}^{I}\right)\right)}{\left(\omega_{t}^{I}-2\right)\left(B \omega_{t}^{I}\right)^{3 / 2}} \\
& -\frac{1}{\sqrt{\tau}} \frac{\left\|\sigma^{c}(\chi, t)\right\|\left(1-\omega_{t}^{I}\right)\left(B v \omega_{t}^{I^{2}}-\sqrt{B \omega_{t}^{I}}\left(2 N+\left(\omega_{t}^{I}-2\right)\left(v^{2} \omega_{t}^{I}-2 \log (1-\beta)\right)\right)\right)}{B\left(\omega_{t}^{I}-2\right)^{2} \omega_{t}^{I}}
\end{aligned}
$$


To obtain the price dynamics we can use (D.53). By inspection of (D.53) we see that we need to calculate $d \omega_{t}^{I}$ only up to $O(\gamma, \tau)$, since the expression involving $\omega$-terms is pre-multiplied by the first-order difference $\gamma_{I}-\gamma_{I I}$. Thus,

$$
d \omega_{t}^{I}=\mu_{0}^{\omega c}(\chi, t) d t+\sigma_{0}^{\omega c}(\chi, t) d Z_{t}^{e}+O(\gamma, \tau)
$$

where

$$
\begin{aligned}
\mu_{0}^{\omega c}(\chi, t)= & \frac{\omega_{t}^{I}\left(1-\omega_{t}^{I}\right)^{2}}{\sigma_{e}\left(X_{t}\right)^{2} C^{3} A_{t}}\left(A_{t}\left(E C^{2}\left(C+v \sigma_{e}\left(X_{t}\right)\right)-D^{2} v \sigma_{e}\left(X_{t}\right)\right)-2 C^{2} \sigma_{e}\left(X_{t}\right)^{2}\left(C+v \sigma_{e}\left(X_{t}\right)\right)\right) \\
& +\frac{\omega_{t}^{I}\left(1-\omega_{t}^{I}\right)^{2}}{\sigma_{e}\left(X_{t}\right)^{2} \tau}\left(\varrho_{\tau, 0}^{2}-2 v \varrho_{\tau, 0} \sigma_{e}\left(X_{t}\right)+2 \sigma\left(X_{t}\right)\left(v\left(C+v \sigma\left(X_{t}\right)\right)-\sigma_{e}\left(X_{t}\right) \log (1-\beta)\right)\right) \\
& +\frac{2\left(C+v \sigma_{e}\left(X_{t}\right)\right) \omega_{t}^{I}\left(1-\omega_{t}^{I}\right)^{2}}{C \sigma_{e}\left(X_{t}\right)^{2} \sqrt{\tau}} D \\
\sigma_{0}^{\omega c}(\chi, t)= & -\frac{\omega_{t}^{I}\left(1-\omega_{t}^{I}\right)}{\sigma_{e}\left(X_{t}\right)}\left(\frac{D}{C}+\frac{C+v \sigma_{e}\left(X_{t}\right)}{\sqrt{\tau}}\right)
\end{aligned}
$$

with

$$
\begin{aligned}
C= & \sqrt{\varrho_{\tau, 0}^{2}-2 v \varrho_{\tau, 0} \sigma_{e}\left(X_{t}\right)+\sigma_{e}\left(X_{t}\right)^{2}\left(v^{2}-2 \log (1-\beta)\right)}, \\
D= & \sigma_{e}\left(X_{t}\right)^{2}\left(\varrho_{\tau, 0}+\left(v \sigma_{e}\left(X_{t}\right)-\varrho_{\tau, 0}\right)\left(1-\frac{v\left(1-\omega_{t}^{I}\right)}{\sqrt{\omega_{t}^{I} B}}\right)\right), \\
E= & \sigma_{e}\left(X_{t}\right)^{4}\left(1-\frac{v\left(1-\omega_{t}^{I}\right)}{\sqrt{\omega_{t}^{I} B}}\right)^{2}+2 \sigma_{e}\left(X_{t}\right)^{2}\left(\delta+\mu_{e}\left(X_{t}\right)+\sigma_{e}\left(X_{t}\right)^{2}\left(1-\frac{v\left(1-\omega_{t}^{I}\right)}{\sqrt{\omega_{t}^{I} B}}\right)\right) \\
& +2 \varrho_{\tau, 1}\left(\varrho_{\tau, 0}-v \sigma_{e}\left(X_{t}\right)\right) .
\end{aligned}
$$

We note that the $d Z_{t}^{X}$ term for the dynamics of $\omega_{t}^{I}$ vanishes in $O\left(\gamma^{2}, \tau^{2}\right)$. To obtain the price dynamics, we go back to equation (D.53). Moving from the unconstrained to the constrained economy adds more terms, namely

$$
\begin{aligned}
& \left(\gamma_{I}-\gamma_{I I}\right) A_{t} \xi_{t}\left(d \omega_{t}^{I}+\sigma_{e}\left(X_{t}\right) d\left\langle Z^{e}, \omega^{I}\right\rangle_{t}\right)+\left(\gamma_{I}-\gamma_{I I}\right) A_{t} d\left\langle\xi, \omega^{I}\right\rangle_{t}+O\left(\gamma^{2}, \tau^{2}\right) \\
= & \left(\gamma_{I}-\gamma_{I I}\right) A_{t} \xi_{t}\left(\left(\mu_{0}^{\omega c}(\chi, t)+\left(\rho_{e X} \sigma_{X}\left(X_{t}\right) \frac{\partial \xi_{t} / \partial X}{\xi_{t}}+\sigma_{e}\left(X_{t}\right)\right) \sigma_{0}^{\omega c}(\chi, t)\right) d t+\sigma_{0}^{\omega c}(\chi, t) d Z^{e}\right) \\
& +O\left(\gamma^{2}, \tau^{2}\right) .
\end{aligned}
$$

In the unconstrained economy, all terms of order $O\left(\gamma^{2}, \tau^{2}\right)$ can be omitted, but this is not true in the constrained economy. Incorporating this term into the price dynamics, and noting from (D.53) that the 
price dynamics must take the form

$$
\frac{d P_{t}+e_{t} d t}{P_{t}}=\alpha^{c}(\chi, t) d t+O(\gamma) d Z_{t}+\sigma_{e}\left(X_{t}\right) d Z_{t}^{e}
$$

we obtain the drift and volatility as claimed in the proposition with

$$
a(\chi, t)=A_{t} \xi_{t}\left(\mu_{0}^{\omega c}(\chi, t)+\left(\rho_{e X} \sigma_{X}\left(X_{t}\right) \frac{\partial \log \xi_{t}}{\partial X}+\sigma_{e}\left(X_{t}\right)\right) \sigma_{0}^{\omega c}(\chi, t)\right) .
$$

Inserting these expressions together with $r^{c}(\chi, t)$ into the dynamics of $\omega_{t}^{I}$, we obtain the cross-sectional wealth dynamics as

$$
\begin{aligned}
d \omega_{t}^{I}= & \left(\mu_{0}^{\omega c}(\chi, t)+\mu_{\gamma}^{\omega c}(\chi, t)+\mu_{\tau}^{\omega c}(\chi, t)\right) d t+\sigma_{0}^{\omega c}(\chi, t) d Z_{t}^{e} \\
& +\left(\sigma_{\tau}^{\omega c}(\chi, t)+\sigma_{\gamma}^{\omega c}(\chi, t)\right)^{\top} d Z_{t}+O\left(\gamma^{2}, \tau^{2}\right),
\end{aligned}
$$

where $\left(\mu_{.}^{\omega c}(\chi, t), \sigma_{\cdot}^{\omega c}(\chi, t)\right)$ are functions of the state variables and the regulatory parameters. Since these functions are given by lengthy expressions, they are not displayed here but can be obtained from the authors on request. 


\section{References}

Anderson, T., L. Benzoni, and J. Lund, 2002, "An Empirical Investigation of Continuous-Time Equity Return Models," Journal of Finance, 57(3), 1239-1284.

Artzner, P., F. Delbaen, J. Eber, and D. Heath, 1999, "Coherent Measures of Risk," Mathematical Finance, 9, 203-228.

Basak, S., and A. Shapiro, 2001, "Value-at-Risk-Based Risk Management: Optimal Policies and Asset Prices," Review of Financial Studies, 14(2), 371-405.

Cochrane, J., 1997, "Where is the Market Going? Uncertain Facts and Novel Theories," Economic Perspectives, Federal Reserve Bank of Chicago, XXI(6).

Cuoco, D., H. He, and S. Issaenko, 2001, "Optimal Dynamic Trading Strategies with Risk Limits," Working paper, Wharton School, University of Pennsylvania.

Cuoco, D., and H. Liu, 2002, "An Analysis of VaR-Based Capital Requirements," Working paper, Wharton School, University of Pennsylvania.

Cvitanic, J., L. Goukasian, and F. Zapatero, 2003, "Monte Carlo simulation of optimal portfolios in complete markets," Journal of Economic Dynamic and Control, 27(6), 971-986.

Danielsson, J., H.-S. Shin, and J.-P. Zigrand, 2001, "Asset Price Dynamics with Value-at-Risk Constrained Traders," Working paper, Department of Accounting and Finance, and Financial Markets Group, London School of Economics.

Detemple, J., R. Garcia, and M. Rindisbacher, 2003, "A Monte Carlo Method for Optimal Portfolios," Journal of Finance, 58, 401-446.

Heston, S., 1993, "A Closed-Form Solution for Options with Stochastic Volatility with Applications to Bond and Currency Options," Review of Financial Studies, 6(2), 341-359. 
Judd, K. L., 1996, "Approximation, Perturbation, and Projection Solution Methods in Economics," in Handbook of Computational Economics. North Holland.

Kogan, L., and R. Uppal, 2001, "Risk aversion and optimal portfolio policies in partial and general equilibrium economies," Working paper, University of British Columbia, Vancouver.

Merton, R. C., 1993, "Optimal Investment Strategies for University Endowment Funds," in Continous-Time Finance. Blackwell, 3 edn., chap. 21, pp. 649-674.

RiskMetrics, 1999, Technical Manual. RiskMetrics Group.

Trojani, F., and P. Vanini, 2002, "Perturbative Solutions of Hamilton Jacobi Bellman Equations in Robust Decision Making," NCCR Working Paper 67, National Center of Competence in Research. 


\section{Notes}

${ }^{1}$ Explicit expressions for higher-order $g$-functions can be obtained from the authors.

${ }^{2}$ See, e.g., Merton (1993) for the interpretation of intermediate consumption in a different institutional context.

${ }^{3}$ For brevity we write $w(X, t)$ instead of $w\left(X_{t}, t\right)$. No confusion should occur.

${ }^{4}$ See, e.g., Basak and Shapiro (2001), Cuoco, He, and Issaenko (2001) and Danielsson, Shin, and Zigrand (2001).

${ }^{5}$ By $\mathbb{1}_{\mathcal{A}}$ we denote the Heaviside function which equals one if $\mathcal{A}$ is true and zero otherwise. 